\title{
Minimax Design of Two-Channel Nonuniform-Division Filterbanks Using IIR Allpass Filters
}

\author{
Ju-Hong Lee and Yuan-Hau Yang
}

\begin{abstract}
The design of two-channel linear-phase nonuniform-division filter (NDF) banks constructed by infinite impulse response (IIR) digital allpass filters (DAFs) in the sense of $L_{\infty}$ error criteria is considered. First, the theory of two-channel NDF bank structures using two IIR DAFs is developed. Then, the design problem is appropriately formulated to result in a simple optimization problem. Utilizing a variant of Karmarkar's algorithm, we can efficiently solve the optimization problem through a frequency sampling and iterative approximation method to find the coefficients for the IIR DAFs. The resulting two-channel NDF banks can possess approximately linear-phase response without magnitude distortion. The effectiveness of the proposed technique is achieved by forming an appropriate Chebyshev approximation of a desired phase response and then to find its solution from a linear subspace in a few iterations. Several simulation examples are presented for illustration and comparison.
\end{abstract}

Index Terms-Allpass filter, minimax optimization, nonuniformdivision filterbank.

\section{INTRODUCTION}

$\mathbf{F}$ OR many communication and signal processing systems, quadrature mirror filter (QMF) banks have been widely used to achieve the goals of subband coding and short-time spectral analysis [1]-[4]. In these applications, a QMF bank is used to decompose a signal into subbands and the subband signals in the analysis system are decimated by an integer equal to the number of subbands. However, two-channel QMF banks are not appropriate systems to match the needs in a great variety of applications. A typical example is critical band analysis with a filterbank that can be utilized in spectral analysis, coding, enhancement, speech recognition, and audio signals. For the subband coding of speech and audio signals, the most appropriate decomposition must consider the critical bands of the ear. It has been mentioned in [5] that these critical bands have nonuniform bandwidths and cannot be easily constructed by conventional tree structure based on two-channel QMF banks. Hence, it is worth exploiting the design problem of two-channel nonuniform-division filter (NDF) banks with linear phase characteristics.

Manuscript received August 20, 2003; revised November 12, 2003. This work was supported by the National Science Council under Grant NSC91-2219-E002-046. The associate editor coordinating the review of this paper and approving it for publication was Dr. Xiang-Gen Xia.

The authors are with the Department of Electrical Engineering, National Taiwan University, Taipei 106, Taiwan, R.O.C. (e-mail: juhong@cc.ee.ntu.edu.tw).

Digital Object Identifier 10.1109/TSP.2004.836532
In the literature, the authors in [6] have presented the basic theory regarding the principle and related conditions of perfect reconstruction for NDF banks. Methods for designing the NDF banks were also proposed in [6]. However, solving the resulting design problem with nonlinear constraints is not an easy task. A different structure for NDF banks was introduced in [7], and a design method based on the use of pseudo-QMF was also presented. The main drawback is that the resulting NDF bank requires FIR filters with complex coefficients to reduce the aliasing distortion. Recently, one of the authors proposed a structure for two-channel NDF banks and presented design methods for optimally designing FIR NDF banks using the least-absolute $\left(L_{1}\right)$ error [8], [9], least-squares $\left(L_{2}\right)$ error [10], [11], and minimax $\left(L_{\infty}\right)$ error criteria [12], [13], respectively.

Although the NDF bank designs in [9], [11] were developed using IIR analysis filters, all of the designed NDF banks suffer from the magnitude distortion as well as the phase distortion. To alleviate the magnitude distortion, the design results for IIR linear-phase QMF banks based on real allpass sections and complex allpass sections have been reported in [14]-[17], and [18] and [19], respectively. The main advantage of using allpass sections is that the designed IIR QMF banks can possess approximately linear phase response without magnitude distortion.

In this paper, a technique for the minimax design of twochannel linear-phase NDF banks using real IIR digital allpass filters (DAFs) is presented. We first develop the principle and structure for the NDF banks using real IIR DAFs. Then, the design problem is formulated by using the minimax error criteria on the phase approximation to obtain an appropriate objective function that leads to a nonlinear optimization problem. To effectively tackle the resulting nonlinear minimax optimization problem, we utilize a nonlinear minimax algorithm [20] to result in a sequence of linear Chebyshev approximation problems. Each of the linear Chebyshev approximations provides the required increment for updating the filter coefficients during each iteration. As a result, the key operation of the proposed technique is to find the linear Chebyshev approximation of a desired phase response from a linear subspace related to the objective function. This can be easily solved by using a variant of Karmarkar's algorithm [21]. Several design examples showing the effectiveness of the proposed technique are also provided.

This paper is organized as follows. Section II presents the principle of a two-channel linear-phase NDF bank using real IIR DAFs. In Section III, we formulate the associated design problem to obtain a nonlinear minimax optimization problem. 


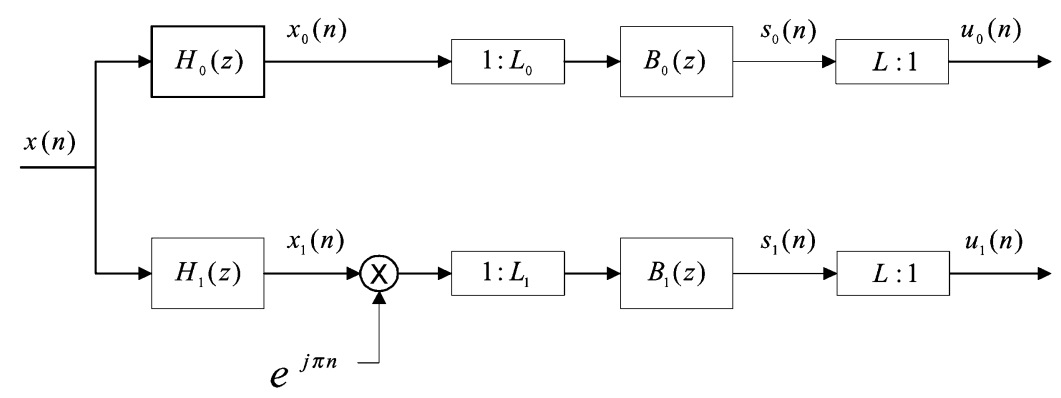

1(a)

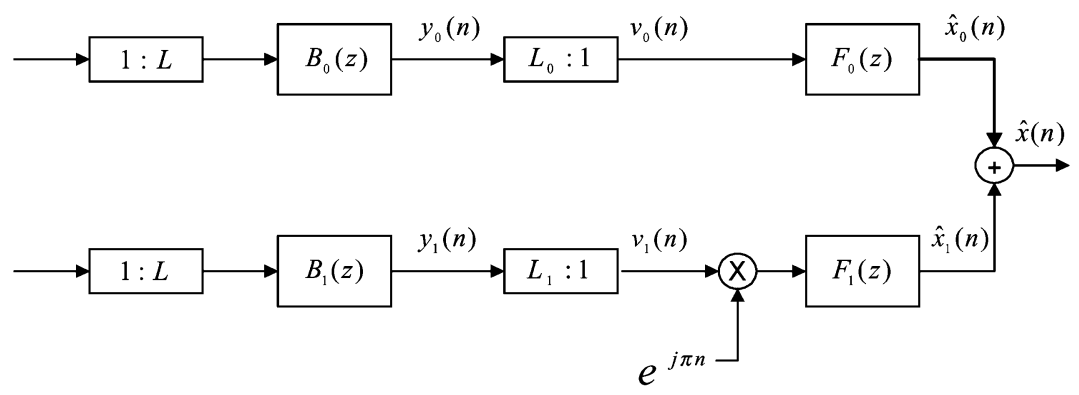

1(b)

Fig. 1. Two-channel nonuniform-division filterbank system. (a) Analysis system. (b) Synthesis system.

A design technique based on a nonlinear minimax algorithm in conjunction with a variant of Karmarkar's algorithm is then developed to solve the resulting optimization problem in Section IV. Section V presents several design examples for illustration and comparison. Finally, we conclude the paper in Section VI.

\section{TWO-CHANNEL LINEAR-PHASE NDF BANKS USING IIR DAF}

\section{A. Conventional Two-Channel NDF Bank Structure}

Consider the two-channel NDF bank with a system architecture shown in Fig. 1 [8]. $H_{0}(\mathrm{z})$ and $H_{1}(\mathrm{z})$ designate the lowpass and highpass analysis filters, respectively, and $F_{0}(\mathrm{z})$ and $F_{1}(\mathrm{z})$ designate the lowpass and highpass synthesis filters, respectively. $B_{0}(\mathrm{z})$ and $B_{1}(\mathrm{z})$ are two lowpass filters that are responsible for achieving aliasing-free operation during the rational decimation and interpolation. Using the modulations of multiplying $\exp (j n \pi)$ in a highpass subband channel leads to the favorable result that $B_{1}(\mathrm{z})$ can be lowpass filter with real coefficients. The desired magnitude responses for $H_{0}(\mathrm{z})$ and $H_{1}(\mathrm{z})$ with passband bandwiths equal to $\pi L_{0} / L$ and $\pi L_{1} / L$, respectively, are shown in Fig. 2 , where $L=L_{0}+L_{1} \cdot \omega_{p}$ and $\omega_{s}$ denote the related band-edge frequencies satisfying $\omega_{p}+\omega_{s}=2 \pi L_{0} / L$. Let the associated magnitude responses be set to

$$
\begin{aligned}
& B_{0}(\omega)= \begin{cases}1, & \omega \in\left[0, \frac{\omega_{s}}{L_{0}}\right] \\
0, & \omega \in\left[\frac{2 \pi-\omega_{s}}{L_{0}}, \pi\right]\end{cases} \\
& B_{1}(\omega)= \begin{cases}1, & \omega \in\left[0, \frac{\pi-\omega_{p}}{L_{1}}\right] \\
0, & \omega \in\left[\frac{\pi+\omega_{p}}{L_{1}}, \pi\right] .\end{cases}
\end{aligned}
$$

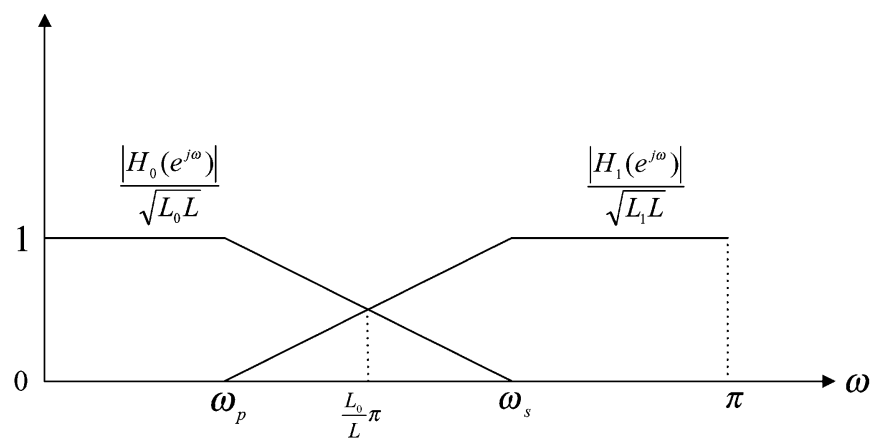

Fig. 2. Desired magnitude specifications for the analysis filters.

$H_{0}(\mathrm{z})$ and $H_{1}(\mathrm{z})$ have zero stopband response. Considering the design of linear-phase lowpass filters $B_{0}(\mathrm{z})$ and $B_{1}(\mathrm{z})$, many effective design techniques can be utilized in the literature, e.g., the well-known Parks-McClellan technique can provide very satisfactory design results. Therefore, we focus on the problem of designing the analysis filters $H_{0}(\mathrm{z})$ and $H_{1}(\mathrm{z})$ of the proposed NDF bank. As shown in [10], the input/output relationship of the NDF bank in the frequency domain is given by

$$
\begin{aligned}
\hat{X}\left(e^{j \omega}\right)= & \frac{e^{-j G_{0} \omega}}{L L_{0}}\left[X\left(e^{j \omega}\right) H_{0}\left(e^{j \omega}\right)\right. \\
& +X\left(e^{j \omega} W_{L}^{L_{0}}\right) H_{0}\left(e^{j \omega} W_{L}^{L_{0}}\right) \\
& \left.+X\left(e^{j \omega} W_{L}^{-L_{0}}\right) H_{0}\left(e^{j \omega} W_{L}^{-L_{0}}\right)\right] F_{0}\left(e^{j \omega}\right) \\
& +\frac{e^{-j G_{1} \omega}}{L L_{1}}\left[X\left(e^{j \omega}\right) H_{1}\left(e^{j \omega}\right)\right. \\
& +X\left(e^{j \omega} W_{L}^{L_{1}}\right) H_{1}\left(e^{j \omega} W_{L}^{L_{1}}\right) \\
& \left.+X\left(e^{j \omega} W_{L}^{-L_{1}}\right) H_{1}\left(e^{j \omega} W_{L}^{-L_{1}}\right)\right] F_{1}\left(e^{j \omega}\right)
\end{aligned}
$$


where $G_{0}$ and $G_{1}$ are the resulting group delays of the upper and lower channels, respectively. $W_{L}=\exp (-j 2 \pi / L)$. Substituting $L=L_{0}+L_{1}, F_{0}\left(e^{j \omega}\right)=H_{0}\left(e^{j \omega}\right), F_{1}\left(e^{j \omega}\right)=$ $-H_{1}\left(e^{j \omega}\right)$ into (2) yields

$$
\begin{aligned}
\hat{X}\left(e^{j \omega}\right)=T\left(e^{j \omega}\right) X\left(e^{j \omega}\right)+ & V_{1}\left(e^{j \omega}\right) X\left(e^{j \omega}\right) \\
& +V_{2}\left(e^{j \omega}\right) X\left(e^{j \omega} W_{L}^{L_{1}}\right)
\end{aligned}
$$

where

$$
\begin{aligned}
T\left(e^{j \omega}\right)= & \frac{e^{-j G_{0} \omega}}{L L_{0}} H_{0}^{2}\left(e^{j \omega}\right)-\frac{e^{-j G_{1} \omega}}{L L_{1}} H_{1}^{2}\left(e^{j \omega}\right) \\
V_{1}\left(e^{j \omega}\right)= & \frac{e^{-j G_{0} \omega}}{L L_{0}} H_{0}\left(e^{j \omega}\right) H_{0}\left(e^{j \omega} W_{L}^{L_{0}}\right) \\
& -\frac{e^{-j G_{1} \omega}}{L L_{1}} H_{1}\left(e^{j \omega}\right) H_{1}\left(e^{j \omega} W_{L}^{L_{0}}\right) \\
V_{2}\left(e^{j \omega}\right)= & \frac{e^{-j G_{0} \omega}}{L L_{0}} H_{0}\left(e^{j \omega}\right) H_{0}\left(e^{j \omega} W_{L}^{L_{1}}\right) \\
& -\frac{e^{-j G_{1} \omega}}{L L_{1}} H_{1}\left(e^{j \omega}\right) H_{1}\left(e^{j \omega} W_{L}^{L_{1}}\right) .
\end{aligned}
$$

The first term of (3) represents the response of a linear shiftinvariant system $T\left(e^{j \omega}\right)$ with input $X\left(e^{j \omega}\right)$, whereas the other two terms represent the resulting aliasing distortion. Therefore, the perfect reconstruction for a group delay $g_{d}$ requires the following conditions:

$$
\begin{aligned}
& \text { PR1: } T\left(e^{j \omega}\right)=e^{-j g_{d} \omega}, \quad \forall \omega \in[0,2 \pi] \\
& \text { PR2: } V_{1}(\omega)=0, \quad \forall \omega \in\left[\omega_{p}, \omega_{s}\right] \\
& \text { PR3: } V_{2}(\omega)=0, \quad \forall \omega \in\left[2 \pi-\omega_{s}, 2 \pi-\omega_{p}\right] .
\end{aligned}
$$

Assuming $G_{0}=G_{1}$ for simplicity, we can neglect the phase term $e^{-j \omega G 0}$ of (4) and express $T\left(e^{j \omega}\right)$ as

$$
\hat{T}\left(e^{j \omega}\right)=\frac{H_{0}^{2}\left(e^{j \omega}\right)}{L L_{0}}-\frac{H_{1}^{2}\left(e^{j \omega}\right)}{L L_{1}} .
$$

Moreover, we note from (5) and (6) and $L=L_{0}+L_{1}$ that the aliasing distortion can be eliminated if

$$
\begin{aligned}
\hat{V}\left(e^{j \omega}\right)=\frac{1}{L L_{0}} H_{0}\left(e^{j\left(\omega-\omega_{p}-\omega_{s}\right)}\right) H_{0}\left(e^{j \omega}\right) & \\
& -\frac{1}{L L_{1}} H_{1}\left(e^{j\left(\omega-\omega_{p}-\omega_{s}\right)}\right) H_{1}\left(e^{j \omega}\right)
\end{aligned}
$$

equals zero for $\omega_{p} \leqq \omega \leqq \omega_{s}$. Following (8) and (9), we can reformulate the conditions of (7) for perfect reconstruction as follows:

$$
\begin{aligned}
& \text { PR1 }: \hat{T}\left(e^{j \omega}\right)=e^{-j k_{d} \omega}, \quad \text { for } 0 \leq \omega \leq \pi \\
& \text { PR2 }: H_{0}\left(e^{j \omega}\right)=0, \quad \text { for } \omega_{s} \leq \omega \leq \pi \\
& \text { PR3 }: H_{1}\left(e^{j \omega}\right)=0, \quad \text { for } 0 \leq \omega \leq \omega_{p} \\
& \text { PR4 }: \frac{1}{L L_{0}} H_{0}\left(e^{j\left(\omega-\omega_{p}-\omega_{s}\right)}\right) H_{0}\left(e^{j \omega}\right) \\
& \quad=\frac{1}{L L_{1}} H_{1}\left(e^{j\left(\omega-\omega_{p}-\omega_{s}\right)}\right) H_{1}\left(e^{j \omega}\right), \quad \text { for } \omega_{p} \leq \omega \leq \omega_{s}
\end{aligned}
$$

where $k_{d}=g_{d}-G_{0}$.

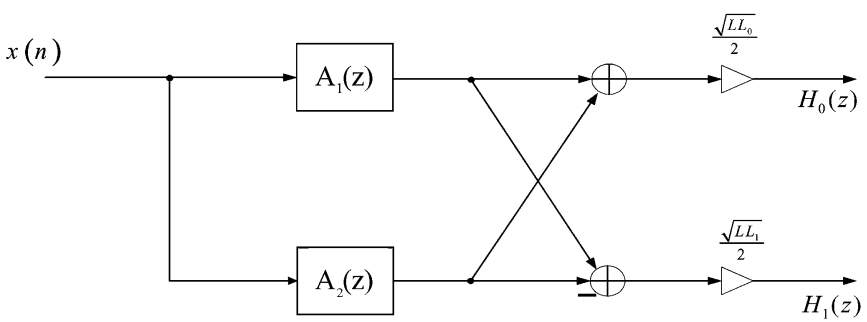

Fig. 3. Proposed system structure for the analysis filters.

\section{B. IIR DAF Based Two-Channel NDF Bank Structure}

Let the frequency responses for two IIR DAFs be expressed as

$$
\begin{aligned}
A_{1}\left(e^{j \omega}\right) & =e^{-j N_{1} \omega} \frac{\sum_{n=0}^{N_{1}} a_{1}(n) e^{j n \omega}}{\sum_{n=0}^{N_{1}} a_{1}(n) e^{-j n \omega}} \\
& =\frac{e^{-j N_{1} \omega} D_{1}^{*}\left(e^{j \omega}\right)}{D_{1}\left(e^{j \omega}\right)}=e^{j \theta_{1}(\omega)}
\end{aligned}
$$

and

$$
\begin{aligned}
A_{2}\left(e^{j \omega}\right) & =e^{-j N_{2} \omega} \frac{\sum_{n=0}^{N_{2}} a_{2}(n) e^{j n \omega}}{\sum_{n=0}^{N_{2}} a_{2}(n) e^{-j n \omega}} \\
& =\frac{e^{-j N_{2} \omega} D_{2}^{*}\left(e^{j \omega}\right)}{D_{2}\left(e^{j \omega}\right)}=e^{j \theta_{2}(\omega)}
\end{aligned}
$$

with orders $N_{1}$ and $N_{2}$, respectively. Using these two IIR DAFs, we construct the lowpass and highpass analysis filters $H_{0}(\mathrm{z})$ and $H_{1}(\mathrm{z})$ of Fig. 1 as follows:

$$
\begin{aligned}
H_{0}\left(e^{j \omega}\right)= & \frac{\sqrt{L L_{0}}}{2}\left[A_{1}\left(e^{j \omega}\right)+A_{2}\left(e^{j \omega}\right)\right] \\
= & \frac{\sqrt{L L_{0}}}{2}\left[e^{j \theta_{1}(\omega)}+e^{j \theta_{2}(\omega)}\right] \\
= & \sqrt{L L_{0}} \cdot \cos \left(\frac{\theta_{1}(\omega)-\theta_{2}(\omega)}{2}\right) \\
& \cdot \exp \left(j \frac{\theta_{1}(\omega)+\theta_{2}(\omega)}{2}\right)
\end{aligned}
$$

and

$$
\begin{aligned}
H_{1}\left(e^{j \omega}\right)= & \frac{\sqrt{L L_{1}}}{2}\left[A_{1}\left(e^{j \omega}\right)-A_{2}\left(e^{j \omega}\right)\right] \\
= & \frac{\sqrt{L L_{1}}}{2}\left[e^{j \theta_{1}(\omega)}-e^{j \theta_{2}(\omega)}\right] \\
= & j \sqrt{L L_{1}} \cdot \sin \left(\frac{\theta_{1}(\omega)-\theta_{2}(\omega)}{2}\right) \\
& \cdot \exp \left(j \frac{\theta_{1}(\omega)+\theta_{2}(\omega)}{2}\right)
\end{aligned}
$$

respectively. Fig. 3 shows the analysis portion of the proposed two-channel NDF bank structure. Substituting (13) and (14) into (8) yields

$$
\begin{aligned}
\hat{T}\left(e^{j \omega}\right)= & \frac{H_{0}^{2}\left(e^{j \omega}\right)}{L L_{0}}-\frac{H_{1}^{2}\left(e^{j \omega}\right)}{L L_{1}} \\
= & \cos ^{2}\left(\frac{\theta_{1}(\omega)-\theta_{2}(\omega)}{2}\right) \cdot e^{j\left[\theta_{1}(\omega)+\theta_{2}(\omega)\right]} \\
& +\sin ^{2}\left(\frac{\theta_{1}(\omega)-\theta_{2}(\omega)}{2}\right) \cdot e^{j\left[\theta_{1}(\omega)+\theta_{2}(\omega)\right]} \\
= & e^{j\left[\theta_{1}(\omega)+\theta_{2}(\omega)\right]}
\end{aligned}
$$




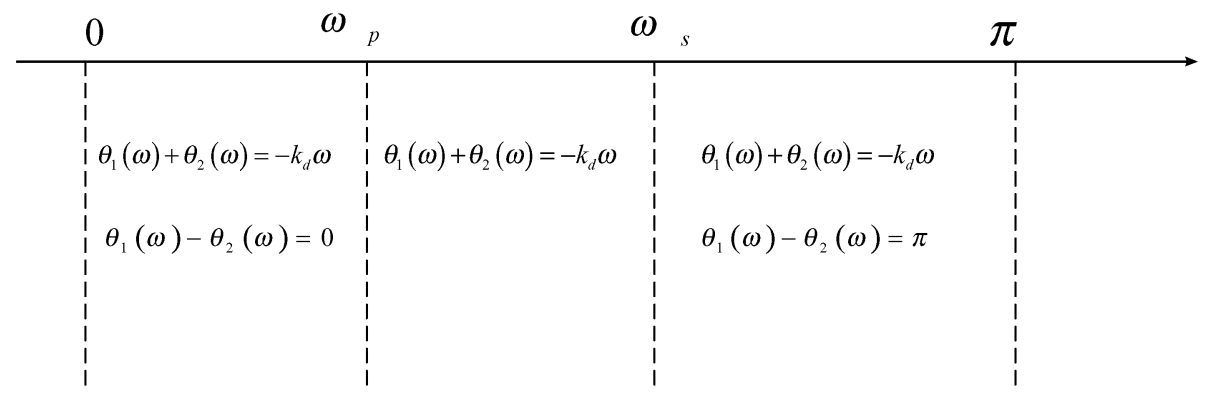

Fig. 4. Constraints on the phase responses of the IIR DAFS.

Equation (15) reveals that the proposed two-channel NDF bank structure possesses perfect magnitude response, i.e., there is no magnitude distortion, and its phase is equal to the sum of the phases of $A_{1}\left(e^{j \omega}\right)$ and $A_{2}\left(e^{j \omega}\right)$. Moreover, comparing PR1 of (10) and (15), we note that the two-channel NDF bank possesses linear-phase response if the following condition is satisfied:

$$
\theta_{1}(\omega)+\theta_{2}(\omega)=-k_{d} \omega, \quad \text { for } 0 \leq \omega \leq \pi
$$

In contrast, the other two conditions PR2 and PR3 in (10) require that the phase responses of (13) and (14) must satisfy the following two conditions:

$$
\begin{aligned}
& \theta_{1}(\omega)-\theta_{2}(\omega)=0, \quad \text { for } 0 \leq \omega \leq \omega_{p} \\
& \theta_{1}(\omega)-\theta_{2}(\omega)=\pi, \quad \text { for } \omega_{s} \leq \omega \leq \pi
\end{aligned}
$$

Fig. 4 depicts the required relationship between the two phase responses $\theta_{1}(\omega)$ and $\theta_{2}(\omega)$ in the frequency band. As to the condition PR4 of (10), we substitute (13) and (14) into the equation shown by PR4 to obtain

$$
\begin{aligned}
& \cos (\left.\frac{\theta_{1}\left(\omega-\omega_{p}-\omega_{s}\right)-\theta_{2}\left(\omega-\omega_{p}-\omega_{s}\right)}{2}\right) \\
& \cdot \exp \left(j \frac{\theta_{1}\left(\omega-\omega_{p}-\omega_{s}\right)+\theta_{2}\left(\omega-\omega_{p}-\omega_{s}\right)}{2}\right) \\
& \cdot \cos \left(\frac{\theta_{1}(\omega)-\theta_{2}(\omega)}{2}\right) \cdot \exp \left(j \frac{\theta_{1}(\omega)+\theta_{2}(\omega)}{2}\right) \\
&=-\sin \left(\frac{\theta_{1}\left(\omega-\omega_{p}-\omega_{s}\right)-\theta_{2}\left(\omega-\omega_{p}-\omega_{s}\right)}{2}\right) \\
& \cdot \exp \left(j \frac{\theta_{1}\left(\omega-\omega_{p}-\omega_{s}\right)+\theta_{2}\left(\omega-\omega_{p}-\omega_{s}\right)}{2}\right) \\
& \cdot \sin \left(\frac{\theta_{1}(\omega)-\theta_{2}(\omega)}{2}\right) \cdot \exp \left(j \frac{\theta_{1}(\omega)+\theta_{2}(\omega)}{2}\right)
\end{aligned}
$$

which can be further simplified as follows:

$$
\begin{aligned}
& \cos \left(\frac{\theta_{1}\left(\omega-\omega_{p}-\omega_{s}\right)-\theta_{2}\left(\omega-\omega_{p}-\omega_{s}\right)}{2}\right) \\
& \cdot \cos \left(\frac{\theta_{1}(\omega)-\theta_{2}(\omega)}{2}\right) \\
& +\sin \left(\frac{\theta_{1}\left(\omega-\omega_{p}-\omega_{s}\right)-\theta_{2}\left(\omega-\omega_{p}-\omega_{s}\right)}{2}\right) \\
& \quad \cdot \sin \left(\frac{\theta_{1}(\omega)-\theta_{2}(\omega)}{2}\right)=0 .
\end{aligned}
$$

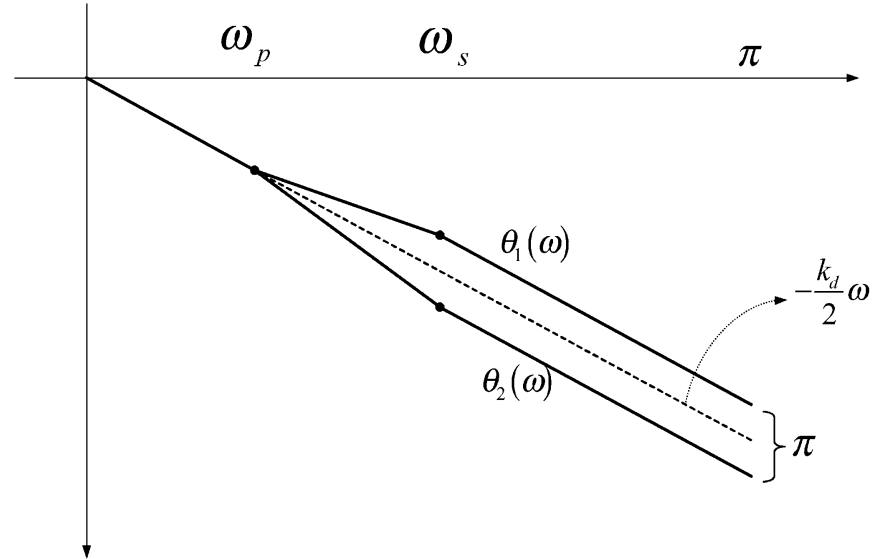

Fig. 5. Proposed design specifications for the phase responses of the IIR DAFs.

Hence, the resulting condition for eliminating aliasing distortion becomes

$$
\begin{array}{r}
\cos \left(\frac{\theta_{1}\left(\omega-\omega_{p}-\omega_{s}\right)-\theta_{2}\left(\omega-\omega_{p}-\omega_{s}\right)}{2}\right. \\
\left.-\frac{\theta_{1}(\omega)-\theta_{2}(\omega)}{2}\right)=0, \text { for } \omega_{p} \leq \omega \leq \omega_{s} .
\end{array}
$$

Based on the above conditions given by (16)-(18) and (21) for the proposed NDF bank, we can impose the ideal phase responses for $\theta_{1}(\omega)$ and $\theta_{2}(\omega)$, respectively, as follows:

$$
\begin{aligned}
P_{1 d}(\omega)= \begin{cases}-\frac{k_{d}}{2} \omega, & \omega \in \Omega_{1} \\
-\frac{k_{d}}{2} \omega+\frac{\omega-\omega_{p}}{\omega_{s}-\omega_{p}} \cdot \frac{\pi}{2}, & \omega \in \Omega_{2} \\
-\frac{k_{d}}{2} \omega+\frac{\pi}{2}, & \omega \in \Omega_{3}\end{cases} \\
\text { and } P_{2 d}(\omega)= \begin{cases}-\frac{k_{d}}{2} \omega, & \omega \in \Omega_{1} \\
-\frac{k_{d}}{2} \omega-\frac{\omega-\omega_{p}}{\omega_{s}-\omega_{p}} \cdot \frac{\pi}{2}, & \omega \in \Omega_{2} \\
-\frac{k_{d}}{2} \omega-\frac{\pi}{2}, & \omega \in \Omega_{3}\end{cases}
\end{aligned}
$$

where $\Omega_{1}=\left[0, \omega_{p}\right], \Omega_{2}=\left(\omega_{p}, \omega_{s}\right)$, and $\Omega_{3}=\left[\omega_{s}, \pi\right]$ represent the three frequency bands in $[0, \pi]$. Fig. 5 plots the ideal phase responses $P_{1 d}(\omega)$ and $P_{2 d}(\omega)$. Equations (22) and (23) reveal that all of the conditions of (16)-(18) and (21) required for perfect reconstruction can be satisfied. Moreover, $P_{1 d}(\omega)$ and $P_{2 d}(\omega)$ satisfy the following stability constraints for the IIR DAFs $A_{i}(\mathrm{z})$ [22], [23]: $\theta_{i}(\omega)$ is monotonically decreasing and $\theta_{i}(\pi)=\theta_{i}(0)-N_{i} \pi$, for $i=1,2$. As a result, the design 
TABLE I

SigNIFICANT DESIGN RESULTS FOR EXAMPLE 1

\begin{tabular}{c|c|c}
\hline & Proposed Technique & Technique of [11] \\
\hline Filter order & 22,23 & $10 / 10,11 / 11$ \\
\hline No. of coefficients & 45 & 44 \\
\hline PRE $(\mathrm{dB})$ & $2.7001 \times 10^{-14}$ & 0.0148 \\
\hline MVGD (samples) & 0.0680 & 0.0583 \\
\hline $\mathrm{NPSR}_{0}(\mathrm{~dB})$ & 33.80 & 32.21 \\
\hline $\mathrm{NPSR}_{1}(\mathrm{~dB})$ & 33.79 & 32.07 \\
\hline $\mathrm{MVPGD}_{0}(\mathrm{samples})$ & 0.0340 & 0.0158 \\
\hline MVPGD $_{1}($ samples$)$ & 0.0338 & 0.0230 \\
\hline MVFBR & $2.28 \times 10^{-3}$ & $2.27 \times 10^{-3}$ \\
\hline No. of iterations & 6 & 37 \\
\hline
\end{tabular}

problem of the proposed two-channel NDF bank of Fig. 3 is finding the real coefficients $a_{i}(n)$ for the IIR DAFs $A_{i}(\mathrm{z})$ such that the conditions listed in (22) and (23) can be approximately met in some optimal sense.

\section{Problem Formulation of Linear-Phase NDF BANK DESIGN}

According to the IIR DAFs $A_{i}(\mathrm{z})$ given by (11) and (12), their phase responses can be expressed as

$$
\theta_{i}(\omega)=-N_{i} \omega-2 \phi_{i}(\omega)
$$

where $\phi_{i}(\omega)$ is given by

$$
\begin{aligned}
\phi_{i}(\omega) & =-\left(N_{i} \omega+\theta_{i}(\omega)\right) / 2 \\
& =-\tan ^{-1}\left[\frac{\sum_{n=1}^{N_{i}} a_{i}(n) \sin (n \omega)}{1+\sum_{n=1}^{N_{i}} a_{i}(n) \cos (n \omega)}\right], \quad i=1,2
\end{aligned}
$$

represents the phase of $D_{i}(\omega)$ in (11) and (12), for $i=1,2$. The coefficients $a_{i}(0)$, for $i=1,2$, are set to one without loss of generality. Substituting (24) and (25) into (15) yields the corresponding phase response of the NDF bank as follows:

$$
\begin{aligned}
\theta_{T}(\omega)= & -\left(N_{1}+N_{2}\right) \omega \\
& -2\left(\tan ^{-1}\left[\frac{-\sum_{n=1}^{N_{1}} a_{1}(n) \sin (n \omega)}{1+\sum_{n=1}^{N_{1}} a_{1}(n) \cos (n \omega)}\right]\right. \\
& \left.+\tan ^{-1}\left[\frac{-\sum_{n=1}^{N_{2}} a_{2}(n) \sin (n \omega)}{1+\sum_{n=1}^{N_{2}} a_{2}(n) \cos (n \omega)}\right]\right) .
\end{aligned}
$$

From (16) and (26), we observe that the proposed two-channel NDF bank achieves the perfect reconstruction with group delay $k_{d}=N_{1}+N_{2}$ and without magnitude distortion if the second term of the right-hand side of (26) vanishes, i.e.,

$$
\phi_{1}(\omega)+\phi_{2}(\omega)=0 .
$$

As to the phase responses of the $D_{i}(\omega)$, the conditions shown by (22) and (23) lead to the following conditions on $\phi_{i}(\omega)$ to be satisfied for perfect reconstruction

$$
P_{i}(\omega)=-\left(N_{i} \omega+P_{\text {id }}(\omega)\right) / 2, \quad i=1,2 .
$$

Utilizing the results of (27) and (28), we can formulate the design problem as follows: Finding the real filter coefficients $a_{i}(n)$ of the IIR DAFs $A_{i}(\mathrm{z}), i=1,2$ and $n=1,2, \ldots, N_{i}$, such that the resulting phase responses $\phi_{i}(\omega)$ given by (25) approximate the desired phase responses, as shown by (27) and (28) in the minimax sense. Therefore, the corresponding design problem can be written as

$$
\begin{gathered}
\operatorname{Minimize}\left(\gamma_{1}\left\|\phi_{1}(\omega)-P_{1}(\omega)\right\|+\gamma_{2}\left\|\phi_{2}(\omega)-P_{2}(\omega)\right\|\right. \\
+\gamma \| \tan ^{-1}\left[\frac{\sum_{n=1}^{N_{1}} a_{1}(n) \sin (n \omega)}{1+\sum_{n=1}^{N_{1}} a_{1}(n) \cos (n \omega)}\right] \\
\left.+\tan ^{-1}\left[\frac{\sum_{n=1}^{N_{2}} a_{2}(n) \sin (n \omega)}{1+\sum_{n=1}^{N_{2}} a_{2}(n) \cos (n \omega)}\right] \|\right)
\end{gathered}
$$

where $\|\mathrm{x}\|$ denotes the Chebyshev norm of $\mathrm{x}$. The parameters $\gamma_{1}, \gamma_{2}$, and $\gamma$ represent the relative weights between the three peak error terms. However, we note from (29) that the overall error function to be minimized is a highly nonlinear function of the real filter coefficients $a_{i}(n)$. Directly minimizing (29) is not an easy task.

\section{Proposed Design Technique}

In this section, we present a design technique based on the nonlinear minimax algorithm of [20] in conjunction with a variant of Karmarkar's algorithm [21] for solving the resulting minimization problem of (29). This is through a frequency sampling and iterative approximation scheme to find the optimal coefficients $a_{i}(n), n=1,2, \ldots, N_{i}, i=1,2$ for the real IIR DAFs shown by (11) and (12).

\section{A. Frequency Sampling and Approximation Scheme}

First, we perform some algebraic manipulations for the three peak error terms in (29) to facilitate the design work. Consider the first two peak error terms. Let

$$
M_{i}(\omega)=\frac{\sum_{n=0}^{N_{i}} a_{i}(n) \sin (n \omega)}{1+\sum_{n=1}^{N_{i}} a_{i}(n) \cos (n \omega)} .
$$

Then, the first two peak error terms can be rewritten as

$$
\left\|\phi_{i}(\omega)-P_{i}(\omega)\right\|=\left\|\tan ^{-1}\left(M_{i}(\omega)\right)+\tan ^{-1}\left(P_{i}^{\prime}(\omega)\right)\right\|
$$


TABLE II

DESIGNED FILTER COEFFICIENTS FOR EXAMPLE 1

\begin{tabular}{|c|c|c|}
\hline$n$ & $a_{1}(n)$ & $a_{2}(n)$ \\
\hline 0 & 1.00000000000000 & 1.00000000000000 \\
\hline 1 & 0.15202429367522 & -0.15203828556132 \\
\hline 2 & -0.17780233087694 & 0.20094928817096 \\
\hline 3 & -0.14421913397156 & 0.08660877051942 \\
\hline 4 & 0.02750087580357 & -0.02682925140379 \\
\hline 5 & 0.09177345466284 & -0.03910907417197 \\
\hline 6 & 0.02666614935610 & -0.00457078742727 \\
\hline 7 & -0.03604434209460 & 0.00917970217453 \\
\hline 8 & -0.02780202931437 & 0.00193175606227 \\
\hline 9 & 0.00416094230507 & 0.00075432180727 \\
\hline 10 & 0.00974454465470 & 0.00557452462760 \\
\hline 11 & 0.00108956264399 & 0.00177594704369 \\
\hline 12 & 0.00265042997354 & -0.00691896169754 \\
\hline 13 & 0.00601946712644 & -0.00611130150180 \\
\hline 14 & -0.00229416531232 & 0.00290330847077 \\
\hline 15 & -0.01091878572071 & 0.00680721449532 \\
\hline 16 & -0.00440133350812 & 0.00136846685214 \\
\hline 17 & 0.00919259463296 & -0.00490914269181 \\
\hline 18 & 0.01049162276946 & -0.00423779115900 \\
\hline 19 & -0.00245151896926 & 0.00166545252261 \\
\hline 20 & 0.00125895507680 & -0.00858063228045 \\
\hline 21 & -0.00037087486662 & 0.00119099024719 \\
\hline 22 & & -0.00052504576384 \\
\hline
\end{tabular}

where $P_{i}^{\prime}(\omega)=\tan \left(P_{i}(\omega)\right), i=1,2$. Using the addition formula of inverse tangents, we obtain

$$
\left\|\phi_{i}(\omega)-P_{i}(\omega)\right\|=\left\|\tan ^{-1}\left\{\frac{M_{i}(\omega)+P_{i}^{\prime}(\omega)}{1-M_{i}(\omega) P_{i}^{\prime}(\omega)}\right\}\right\|
$$

Minimizing (32) is equivalent to minimizing the term $Q_{i}(\omega)=$ $\left\|\left(M_{i}(\omega)+P_{i}^{\prime}(\omega)\right) /\left(1-M_{i}(\omega) P_{i}^{\prime}(\omega)\right)\right\|$ since the inverse tangent function is a monotonic function. Plugging (30) into $Q_{i}(\omega)$ and performing some algebraic manipulations on $Q_{i}(\omega)$ yields

$$
\begin{aligned}
& Q_{i}(\omega) \\
& =\left\|\frac{\sum_{n=1}^{N_{i}} a_{i}(n)\left[\sin (n \omega)+P_{i}^{\prime}(\omega) \cdot \cos (n \omega)\right]+P_{i}^{\prime}(\omega)}{\sum_{n=1}^{N_{i}} a_{i}(n)\left[\cos (n \omega)-P_{i}^{\prime}(\omega) \cdot \sin (n \omega)\right]+1}\right\| \\
& i=1,2 .
\end{aligned}
$$

Following the above procedure, we have that minimizing the third peak error term is equivalent to minimizing the following term in (34), shown at the bottom of the page. Consequently, an equivalent minimization problem of (29) can be obtained as follows:

$$
\text { Minimize }\left\{\gamma_{1} Q_{1}(\omega)+\gamma_{2} Q_{2}(\omega)+\gamma R(\omega)\right\} \text {. }
$$

The resulting nonlinear optimization problem given by (35) can be solved through an approximation approach in conjunction with an iterative process as follows. At the $k$ th iteration, we construct the following functions:

$$
\begin{aligned}
& \operatorname{Apx}_{i}\left(\mathbf{a}_{i k}, \omega\right) \\
&=\frac{\sum_{n=1}^{N_{i}} a_{i k}(n)\left[\sin (n \omega)+P_{i}^{\prime}(\omega) \cdot \cos (n \omega)\right]+P_{i}^{\prime}(\omega)}{\sum_{n=1}^{N_{i}} a_{i k}(n)\left[\cos (n \omega)-P_{i}^{\prime}(\omega) \cdot \sin (n \omega)\right]+1} \\
& i=1,2
\end{aligned}
$$

and the $N_{i} \times 1$ gradient vector of $\operatorname{Apx}_{i}\left(\mathbf{a}_{i k}, \omega\right)$ given by

$$
\nabla \operatorname{Apx}_{i}\left(\mathbf{a}_{i k}, \omega\right)=\left[\psi_{i}(\omega, 1) \psi_{i}(\omega, 2) \ldots \psi_{i}\left(\omega, N_{i}\right)\right]^{T}
$$

where $\psi_{i}(\omega, j)$ denotes the $j$ th gradient component of $\operatorname{Apx}_{i}\left(\mathbf{a}_{i k}, \omega\right)$ and is given in (38), shown at the bottom of the next page, for $j=1,2, \ldots, N_{i}$, where $\mathbf{a}_{i k}=$ $\left[a_{i k}(1), a_{i k}(2), \ldots, a_{i k}\left(N_{i}\right)\right]^{\mathrm{T}}$ represents the filter coefficient vector obtained at the $k$ th iteration for the IIR DAFs $A_{i}(\mathrm{z}), i=1,2$. Moreover, we construct (39), shown at the

$$
R(\omega)=\left\|\frac{\left(1+\sum_{n=1}^{N_{2}} a_{2}(n) \cos (n \omega)\right)\left(\sum_{n=1}^{N_{1}} a_{1}(n) \sin (n \omega)\right)+\left(1+\sum_{n=1}^{N_{1}} a_{1}(n) \cos (n \omega)\right)\left(\sum_{n=1}^{N_{2}} a_{2}(n) \sin (n \omega)\right)}{\left(1+\sum_{n=1}^{N_{1}} a_{1}(n) \cos (n \omega)\right)\left(1+\sum_{n=1}^{N_{2}} a_{2}(n) \cos (n \omega)\right)-\left(\sum_{n=1}^{N_{1}} a_{1}(n) \sin (n \omega)\right)\left(\sum_{n=1}^{N_{2}} a_{2}(n) \sin (n \omega)\right) \|}\right\|
$$


bottom of the page, and the $\left(N_{1}+N_{2}\right) \times 1$ gradient vector of $\operatorname{Apx}_{i}\left(\mathbf{a}_{i k}, \omega\right)$, which is given by

$\nabla \operatorname{Apx}_{T}\left(\mathbf{a}_{1 k}, \mathbf{a}_{2 k}, \omega\right)=\left[\psi_{T 1}(\omega, 1) \psi_{T 1}(\omega, 2)\right.$

$$
\left.\ldots \psi_{T 1}\left(\omega, N_{1}\right) \psi_{T 2}(\omega, 1) \ldots \psi_{T 2}\left(\omega, N_{2}\right)\right]^{\mathrm{T}}
$$

where $\psi_{\mathrm{T} 1}(\omega, j)$ denotes the $j$ th gradient component of $\operatorname{Apx}_{T}\left(\mathbf{a}_{1 k}, \mathbf{a}_{2 k}, \omega\right)$ and is given as follows:

$$
\begin{aligned}
\psi_{T_{1}}(\omega, j)= & \frac{\partial \operatorname{Apx}_{T}\left(\mathbf{a}_{1 k}, \mathbf{a}_{2 k}, \omega\right)}{\partial a_{1 k}(j)} \\
= & {\left[\sin (j \omega)\left(1+\sum_{n=1}^{N_{2}} a_{2 k}(n) \cos (n \omega)\right)\right.} \\
& \left.+\cos (j \omega) \sum_{n=1}^{N_{2}} a_{2 k}(n) \sin (n \omega)\right] \\
& \cdot\left[\left(1+\sum_{n=1}^{N_{1}} a_{1 k}(n) \cos (n \omega)\right)\right. \\
& \cdot\left(1+\sum_{n=1}^{N_{2}} a_{2 k}(n) \cos (n \omega)\right) \\
& -\left(\sum_{n=1}^{N_{1}} a_{1 k}(n) \sin (n \omega)\right) \\
& \left.\cdot\left(\sum_{n=1}^{N_{2}} a_{2 k}(n) \sin (n \omega)\right)\right] \\
& -\left[\cos (j \omega)\left(1+\sum_{n=1}^{N_{2}} a_{2 k}(n) \cos (n \omega)\right)\right. \\
& \left.-\sin ^{-1}(j \omega) \sum_{n=1}^{N_{2}} a_{2 k}(n) \sin (n \omega)\right] \\
& \cdot\left[\left(1+\sum_{n=1}^{N_{2}} a_{2 k}(n) \cos (n \omega)\right)\right. \\
& \sum_{n=1}^{N_{1}} a_{1 k}(n) \sin (n \omega)
\end{aligned}
$$

$$
\begin{aligned}
& +\left(1+\sum_{n=1}^{N_{1}} a_{1 k}(n) \cos (n \omega)\right) \\
& \left.\cdot \sum_{n=1}^{N_{2}} a_{2 k}(n) \sin (n \omega)\right] \\
& \cdot\left[\left(1+\sum_{n=1}^{N_{1}} a_{1 k}(n) \cos (n \omega)\right)\right. \\
& \cdot\left(1+\sum_{n=1}^{N_{2}} a_{2 k}(n) \cos (n \omega)\right) \\
& -\left(\sum_{n=1}^{N_{1}} a_{1 k}(n) \sin (n \omega)\right) \\
& \left.\cdot\left(\sum_{n=1}^{N_{2}} a_{2 k}(n) \sin (n \omega)\right)\right]^{-2} .
\end{aligned}
$$

$\psi_{T 2}(\omega, j)$ denotes the $\left(\mathrm{N}_{1}+j\right)$ th gradient component of $\operatorname{Apx}_{T}\left(\mathbf{a}_{1 k}, \mathbf{a}_{2 k}, \omega\right)$ and is given as follows:

$$
\begin{aligned}
\psi_{T_{2}}(\omega, j)= & \frac{\partial \operatorname{Apx}_{T}\left(\mathbf{a}_{1 k}, \mathbf{a}_{2 k}, \omega\right)}{\partial a_{2 k}(j)} \\
= & {\left[\cos (j \omega) \sum_{n=1}^{N_{1}} a_{1 k}(n) \sin (n \omega)\right.} \\
& \left.+\sin (j \omega)\left(1+\sum_{n=1}^{N_{1}} a_{1 k}(n) \cos (n \omega)\right)\right] \\
& \cdot\left[\left(1+\sum_{n=1}^{N_{1}} a_{1 k}(n) \cos (n \omega)\right)\right. \\
& \cdot\left(1+\sum_{n=1}^{N_{2}} a_{2 k}(n) \cos (n \omega)\right) \\
& -\left(\sum_{n=1}^{N_{1}} a_{1 k}(n) \sin (n \omega)\right)
\end{aligned}
$$

$$
\begin{aligned}
\psi_{i}(\omega, j)= & \frac{\partial \operatorname{Apx}_{i}\left(\mathbf{a}_{i k}, \omega\right)}{\partial \mathbf{a}_{i k}(j)} \\
= & \frac{\left[\sin (j \omega)+P_{i}^{\prime}(\omega) \cos (j \omega)\right] \cdot\left(\sum_{n=1}^{N_{i}} a_{i k}(n)\left[\cos (n \omega)-P_{i}^{\prime}(\omega) \sin (n \omega)\right]+1\right)}{\left(\sum_{n=1}^{N_{i}} a_{i k}(n)\left[\cos (n \omega)-P_{i}^{\prime}(\omega) \sin (n \omega)\right]+1\right)^{2}} \\
& -\frac{\left[\cos (j \omega)-P_{i}^{\prime}(\omega) \sin (j \omega)\right] \cdot\left(\sum_{n=1}^{N_{i}} a_{i k}(n)\left[\sin (n \omega)+P_{i}^{\prime}(\omega) \cos (n \omega)\right]+P_{i}^{\prime}(\omega)\right)}{\left(\sum_{n=1}^{N_{i}} a_{i k}(n)\left[\cos (n \omega)-P_{i}^{\prime}(\omega) \sin (n \omega)\right]+1\right)^{2}}, i=1,2
\end{aligned}
$$

$$
\begin{aligned}
& \operatorname{Apx}_{T}\left(\mathbf{a}_{1 k}, \mathbf{a}_{2 k}, \omega\right) \\
& \quad=\frac{\left(1+\sum_{n=1}^{N_{2}} a_{2 k}(n) \cos (n \omega)\right) \sum_{n=1}^{N_{1}} a_{1 k}(n) \sin (n \omega)+\left(1+\sum_{n=1}^{N_{1}} a_{1 k}(n) \cos (n \omega)\right) \sum_{n=1}^{N_{2}} a_{2 k}(n) \sin (n \omega)}{\left(1+\sum_{n=1}^{N_{1}} a_{1 k}(n) \cos (n \omega)\right)\left(1+\sum_{n=1}^{N_{2}} a_{2 k}(n) \cos (n \omega)\right)-\left(\sum_{n=1}^{N_{1}} a_{1 k}(n) \sin (n \omega)\right)\left(\sum_{n=1}^{N_{2}} a_{2 k}(n) \sin (n \omega)\right)}
\end{aligned}
$$




$$
\begin{aligned}
& \left.\cdot\left(\sum_{n=1}^{N_{2}} a_{2 k}(n) \sin (n \omega)\right)\right]^{-1} \\
& -\left[\cos (j \omega)\left(1+\sum_{n=1}^{N_{1}} a_{1 k}(n) \cos (n \omega)\right)\right. \\
& \left.-\sin (j \omega) \sum_{n=1}^{N_{1}} a_{1 k}(n) \sin (n \omega)\right] \\
& \cdot\left[\left(1+\sum_{n=1}^{N_{2}} a_{2 k}(n) \cos (n \omega)\right)\right. \\
& \cdot \sum_{n=1}^{N_{1}} a_{1 k}(n) \sin (n \omega) \\
& +\left(1+\sum_{n=1}^{N_{1}} a_{1 k}(n) \cos (n \omega)\right) \\
& \text { - } \left.\sum_{n=1}^{N_{2}} a_{2 k}(n) \sin (n \omega)\right] \\
& \cdot\left[\left(1+\sum_{n=1}^{N_{1}} a_{1 k}(n) \cos (n \omega)\right)\right. \\
& \cdot\left(1+\sum_{n=1}^{N_{2}} a_{2 k}(n) \cos (n \omega)\right) \\
& \text { - }\left(\sum_{n=1}^{N_{1}} a_{1 k}(n) \sin (n \omega)\right) \\
& \left.\cdot\left(\sum_{n=1}^{N_{2}} a_{2 k}(n) \sin (n \omega)\right)\right]^{-2} \text {. }
\end{aligned}
$$

Let $\Omega_{1 \mathrm{~d}}=\left[\omega_{1}=0, \omega_{2}, \ldots, \omega_{S 1}=\omega_{p}\right], \Omega_{2 \mathrm{~d}}=\left(\omega_{S 1}=\right.$ $\left.\omega_{p}, \omega_{S 1+1}, \ldots \omega_{S 1+S 2+1}=\omega_{s}\right)$, and $\Omega_{3 \mathrm{~d}}=\left[\omega_{S 1+S 2+1}=\right.$ $\left.\omega_{s}, \omega_{S 1+S 2+2}, \ldots, \omega_{S 1+S 2+S 3}=\pi\right]$ represent the three dense grid of frequency bands in $[0, \pi]$. Each of them has grid points uniformly distributed in the individual frequency band. The design process of the proposed technique is then performed on $\Omega_{\mathrm{d}}=\Omega_{1 \mathrm{~d}} \cup \Omega_{2 \mathrm{~d}} \cup \Omega_{3 \mathrm{~d}}$ with $S=S_{1}+S_{2}+S_{3}$ grid points. If the number of grid points is sufficiently large, i.e., $\Omega_{\mathrm{d}}$ is sufficiently close to $\Omega=\{\omega \mid 0 \leq \omega \leq \pi\}$, the obtained best approximation solution of the objective function based on $\Omega_{\mathrm{d}}$ will be close to the best solution found based on $\Omega$. This conclusion can be justified by the theorem due to Cheney [26, ch. 3]. Next, a linearization scheme is utilized to approximate the related phase errors of (36) and (39) due to a perturbation in the filter coefficient vector in the linear subspace spanned by the gradient matrix associated with $\operatorname{Apx}_{i}\left(\mathbf{a}_{i k}, \omega\right)$ and $\operatorname{Apx}_{T}\left(\mathbf{a}_{1 k}, \mathbf{a}_{2 k}, \omega\right)$. As a result, the approximation for minimizing the above peak phase error, as shown in (35), can be formulated as finding the increments $\delta \mathbf{a}_{i}=\left[\delta a_{i k}(1) \delta a_{i k}(2) \ldots \delta a_{i k}\left(N_{i}\right)\right]^{\mathrm{T}}$ of the filter coefficient vectors $\mathbf{a}_{i}$ at the $k$ th iteration such that

$$
\begin{aligned}
& \gamma_{1}\left\|\operatorname{Apx}_{1}\left(\mathbf{a}_{1 k}, \omega_{l}\right)+\delta \mathbf{a}_{1}^{\mathrm{T}} \nabla \operatorname{Apx}_{1}\left(\mathbf{a}_{1 k}, \omega_{l}\right)\right\| \\
& \quad+\gamma_{2}\left\|\operatorname{Apx}_{2}\left(\mathbf{a}_{2 k}, \omega_{l}\right)+\delta \mathbf{a}_{2}^{\mathrm{T}} \nabla \operatorname{Apx}_{2}\left(\mathbf{a}_{2 k}, \omega_{l}\right)\right\| \\
& \quad+\gamma\left\|\operatorname{Apx}_{\mathrm{T}}\left(\mathbf{a}_{1 k}, \mathbf{a}_{2 k}, \omega_{l}\right)+\delta \mathbf{a}_{12}^{\mathrm{T}} \nabla \operatorname{Apx}_{\mathrm{T}}\left(\mathbf{a}_{1 k}, \mathbf{a}_{2 k}, \omega_{l}\right)\right\|
\end{aligned}
$$

is minimized, where $\delta \mathbf{a}_{12}=\left[\begin{array}{ll}\delta \mathbf{a}_{1}^{\mathrm{T}} & \delta \mathbf{a}_{2}^{\mathrm{T}}\end{array}\right]^{\mathrm{T}}$. For details, we can rewrite (43) as follows:

$$
\begin{aligned}
& \gamma_{1}\left\|\operatorname{Apx}_{1}\left(\mathbf{a}_{1 k}, \omega_{l}\right)+\sum_{n=1}^{N_{1}} \delta a_{1 k}(n) \psi_{1}\left(\omega_{l}, n\right)\right\| \\
& +\gamma_{2}\left\|\operatorname{Apx}_{2}\left(\mathbf{a}_{2 k}, \omega_{l}\right)+\sum_{n=1}^{N_{2}} \delta a_{2 k}(n) \psi_{2}\left(\omega_{l}, n\right)\right\| \\
& +\gamma \| \operatorname{Apx}_{\mathrm{T}}\left(\mathbf{a}_{1 k}, \mathbf{a}_{2 k}, \omega_{l}\right)+\sum_{n=1}^{N_{1}} \delta a_{1 k}(n) \psi_{\mathrm{T} 1}\left(\omega_{l}, n\right) \\
& +\sum_{n=1}^{N_{2}} \delta a_{2 k}(n) \psi_{\mathrm{T} 2}\left(\omega_{l}, n\right) \| .
\end{aligned}
$$

We note that the minimization of (44) can be achieved by performing the following minimization problem:

$$
\begin{aligned}
\operatorname{Minimize}\left\{\gamma_{1}\left\|\mathbf{E}_{1} \delta \mathbf{a}_{1}-\mathbf{s}_{1}\right\|+\gamma_{2} \|\right. & \mathbf{E}_{2} \delta \mathbf{a}_{2}-\mathbf{s}_{2} \| \\
& \left.+\gamma\left\|\mathbf{E}_{\mathrm{T}} \delta \mathbf{a}_{12}-\mathbf{s}_{\mathrm{T}}\right\|\right\}
\end{aligned}
$$

where $\mathbf{E}_{1}$ is an $S \times N_{1}$ matrix with the $(i, j)$ th element given by $\mathrm{E}_{1}(i, j)=\psi_{1}\left(\omega_{i}, j\right), \omega_{i} \in \Omega_{\mathrm{d}}, 1 \leqq j \leqq N_{1}, \mathbf{E}_{2}$ is an $S \times N_{2}$ matrix with the $(i, j)$ th element given by $\mathrm{E}_{2}(i, j)=$ $\psi_{2}\left(\omega_{i}, j\right), \omega_{i} \in \Omega_{\mathrm{d}}, 1 \leqq j \leqq N_{2}, \mathbf{s}_{1}$ is an $S \times 1$ column vector with the $i$ th entry given by $s_{1}(i)=-\operatorname{Apx}_{1}\left(\mathbf{a}_{1 k}, \omega_{i}\right), \omega_{i} \in$ $\Omega_{\mathrm{d}}, \mathbf{s}_{2}$ is an $S \times 1$ column vector with the $i$ th entry given by $s_{2}(i)=-\mathrm{Apx}_{2}\left(\mathbf{a}_{2 k}, \omega_{i}\right), \omega_{i} \in \Omega_{\mathrm{d}}, \mathbf{E}_{\mathrm{T}}=\left[\mathbf{E}_{\mathrm{T} 1} \mathbf{E}_{\mathrm{T} 2}\right]$ is an $S \times\left(N_{1}+N_{2}\right)$ matrix with the submatrices $\mathbf{E}_{\mathrm{T} 1}$ and $\mathbf{E}_{\mathrm{T} 2}$ having the $(i, j)$ th elements given by $\mathrm{E}_{\mathrm{T} 1}(i, j)=\psi_{\mathrm{T} 1}\left(\omega_{i}, j\right), \omega_{i} \in \Omega_{\mathrm{d}}$, $1 \leqq j \leqq N_{1}$, and $\mathrm{E}_{\mathrm{T} 2}(i, j)=\psi_{\mathrm{T} 2}\left(\omega_{i}, j\right), \omega_{i} \in \Omega_{\mathrm{d}}, 1 \leqq j \leqq$ $N_{2}$, respectively. Equation (45) represents an equivalent form of the following linear Chebyshev minimization problem:

$$
\begin{aligned}
& \text { Minimize } \gamma_{1} \xi_{1}+\gamma_{2} \xi_{2}+\gamma \xi_{\mathrm{T}} \\
& \text { Subject to }\left\{\begin{array}{l}
\mathbf{E}_{1} \delta \mathbf{a}_{1}-\mathbf{s}_{1} \leq \xi_{1} \mathbf{1} \\
-\mathbf{E}_{1} \delta \mathbf{a}_{1}+\mathbf{s}_{1} \leq \xi_{1} \mathbf{1} \\
\mathbf{E}_{2} \delta \mathbf{a}_{2}-\mathbf{s}_{2} \leq \xi_{2} \mathbf{1} \\
-\mathbf{E}_{2} \delta \mathbf{a}_{2}+\mathbf{s}_{2} \leq \xi_{2} \mathbf{1} \\
\mathbf{E}_{\mathrm{T}} \delta \mathbf{a}_{12}-\mathbf{s}_{\mathrm{T}} \leq \xi_{\mathrm{T}} \mathbf{1} \\
-\mathbf{E}_{\mathrm{T}} \delta \mathbf{a}_{12}+\mathbf{s}_{\mathrm{T}} \leq \xi_{\mathrm{T}} \mathbf{1}
\end{array}\right.
\end{aligned}
$$

where 1 is a $S \times 1$ vector with all entries equal to one. This leads to the standard dual form of a linear programming (LP) problem as follows:

$$
\begin{aligned}
& \text { Maximize } \mathbf{b}^{\mathrm{T}} \mathbf{w} \\
& \text { Subject to } \mathbf{A}^{\mathrm{T}} \mathbf{w} \leqq \mathbf{c}
\end{aligned}
$$

where

$$
\begin{aligned}
\mathbf{A}^{\mathrm{T}} & =\left[\begin{array}{ccccc}
\mathbf{E}_{1} & \mathbf{0}_{2} & \mathbf{- 1} & \mathbf{0} & \mathbf{0} \\
-\mathbf{E}_{1} & \mathbf{0}_{2} & \mathbf{- 1} & \mathbf{0} & \mathbf{0} \\
\mathbf{0}_{1} & \mathbf{E}_{2} & \mathbf{0} & \mathbf{- 1} & \mathbf{0} \\
\mathbf{0}_{1} & -\mathbf{E}_{2} & \mathbf{0} & \mathbf{- 1} & \mathbf{0} \\
\mathbf{E}_{\mathrm{T} 1} & \mathbf{E}_{\mathrm{T} 2} & \mathbf{0} & \mathbf{0} & \mathbf{- 1} \\
-\mathbf{E}_{\mathrm{T} 1} & -\mathbf{E}_{\mathrm{T} 2} & \mathbf{0} & \mathbf{0} & \mathbf{- 1}
\end{array}\right] \\
\mathbf{w} & =\left[\begin{array}{c}
\delta \mathbf{a}_{1} \\
\delta \mathbf{a}_{2} \\
\xi_{1} \\
\xi_{2} \\
\xi_{\mathrm{T}}
\end{array}\right], \quad \mathbf{b}=\left[\begin{array}{c}
\mathbf{0}_{N 1} \\
\mathbf{0}_{N 2} \\
-\gamma_{1} \\
-\gamma_{2} \\
-\gamma
\end{array}\right], \quad \mathbf{c}=\left[\begin{array}{c}
\mathbf{s}_{1} \\
-\mathbf{s}_{1} \\
\mathbf{s}_{2} \\
-\mathbf{s}_{2} \\
\mathbf{s}_{\mathrm{T}} \\
-\mathbf{s}_{\mathrm{T}}
\end{array}\right] .
\end{aligned}
$$


The $\mathbf{0}_{1}$ and $\mathbf{0}_{2}$ in $\mathbf{A}$ are zero matrices with sizes equal to $S \times N_{1}$ and $S \times N_{2}$, respectively, whereas 1 and $\mathbf{0}$ are $S \times 1$ vectors with entries equal to one and zero, respectively. In contrast, $\mathbf{0}_{N 1}$ and $\mathbf{0}_{N 2}$ are zero vectors with sizes equal to $N_{1} \times 1$ and $N_{2} \times 1$, respectively. Using [24, Def. 3], we can easily obtain the standard primal form for (47) as follows:

$$
\begin{aligned}
& \text { Minimize } \mathbf{c}^{\mathbf{T}} \mathbf{x} \\
& \text { Subject to } \mathbf{A x}=\mathbf{b}, \mathbf{x} \geqq 0
\end{aligned}
$$

where $\mathbf{w}$ and $\mathbf{x}=\left[x_{1} x_{2} \ldots x_{6 S}\right]^{\mathrm{T}}$, respectively, denote the dual variable and the corresponding primal variable for the above LP problems. In the following, we describe how to adopt the primalform affine scaling (PAS) variant of Karmarkar's algorithm of [21] for solving (48).

Assume that an initial solution $\mathbf{x}$ that satisfies the constraints is given. Then, $\mathbf{x}$ is mapped into a vector $\mathbf{y}^{0}$ with all entries equal to one as follows:

$$
\mathbf{y}=\mathbf{D}_{\mathrm{x}}^{-1} \mathbf{x}
$$

where $\mathbf{D}_{\mathbf{x}}$ denotes a diagonal matrix containing the entries of $\mathbf{x}$. Based on the mapping, we create

$$
\underline{\mathbf{A}}=\mathbf{A D}_{\mathrm{x}} \quad \text { and } \quad \underline{\mathbf{c}}=\mathbf{D}_{\mathrm{x}} \mathbf{c} .
$$

To satisfy the equality constraints, we project $\underline{\mathbf{c}}$ onto the null space of $\underline{\mathbf{A}}$ to obtain

$$
\underline{\mathbf{c}}_{\mathrm{p}}=\mathbf{P} \underline{\mathbf{c}}
$$

where

$$
\mathbf{P}=\mathbf{I}-\underline{\mathbf{A}}^{\mathrm{T}}\left(\underline{\mathbf{A A}}^{\mathrm{T}}\right)^{-1} \underline{\mathbf{A}}
$$

denotes the projection operator. Next, we move from the initial $\mathbf{y}$ to $\mathbf{y}^{1}$ in the direction $-\underline{\mathbf{c}}_{p}$ to reduce the transformed objective function in the maximum rate according to

$$
\mathbf{y}^{1}=1-\mu \frac{\underline{\mathbf{c}}_{p}}{\max _{\mathrm{i}}\left(\mathbf{e}_{\mathrm{i}}^{\mathrm{T}} \underline{\mathbf{c}}_{\mathrm{p}}\right)}
$$

where the required step size $\mu \in(0,1)$ is chosen so that $\mathbf{y}^{1}>$ $\mathbf{0 , 1}$ represents a vector with appropriate size and all entries equal to one, and $\mathbf{e}_{\mathbf{i}}$ is a vector with appropriate size and the $i$ th entry equal to one and the others equal to zero. After obtaining $\mathbf{y}^{1}$, we then find a new feasible solution $\mathbf{x}^{1}$ for (48) by performing the inverse mapping.

Consider the projection operator given by (52). Substituting (50) into (52) yields

$$
\mathbf{P}=\mathbf{I}-\mathbf{D}_{\mathrm{x}} \mathbf{A}^{\mathrm{T}}\left(\mathbf{A D}_{\mathrm{x}}^{2} \mathbf{A}^{\mathrm{T}}\right)^{-1} \mathbf{A} \mathbf{D}_{\mathrm{x}} .
$$

Accordingly, we have from (50), (51), and (54) that

$$
\underline{\mathbf{c}}_{\mathrm{p}}=\mathbf{D}_{\mathrm{x}}\left[\mathbf{I}-\mathbf{A}^{\mathrm{T}}\left(\mathbf{A D}_{\mathrm{x}}^{2} \mathbf{A}^{\mathrm{T}}\right)^{-1} \mathbf{A D}_{\mathrm{x}}^{2}\right] \mathbf{c} .
$$

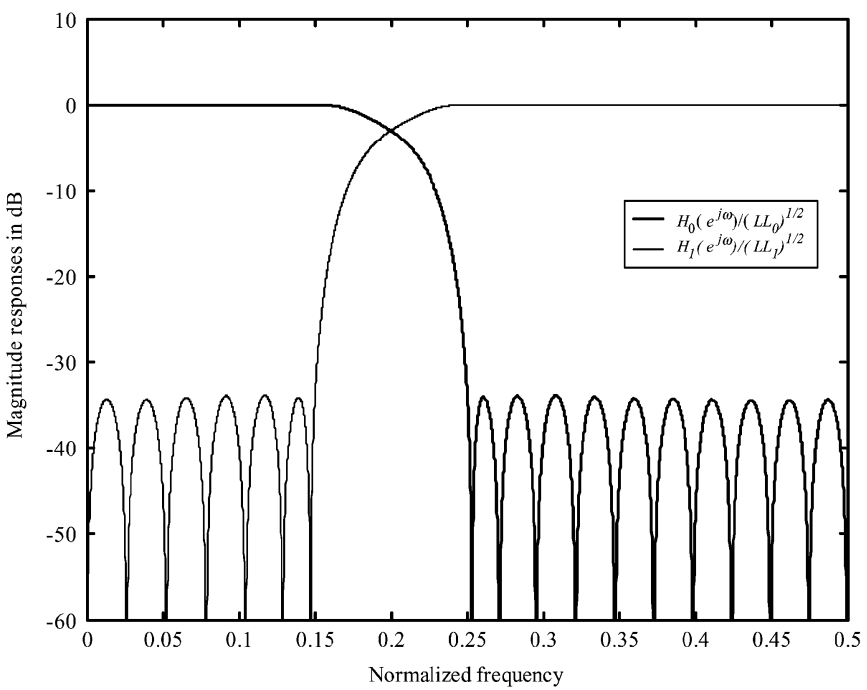

Fig. 6. Magnitude responses of the designed analysis filters for Example 1.

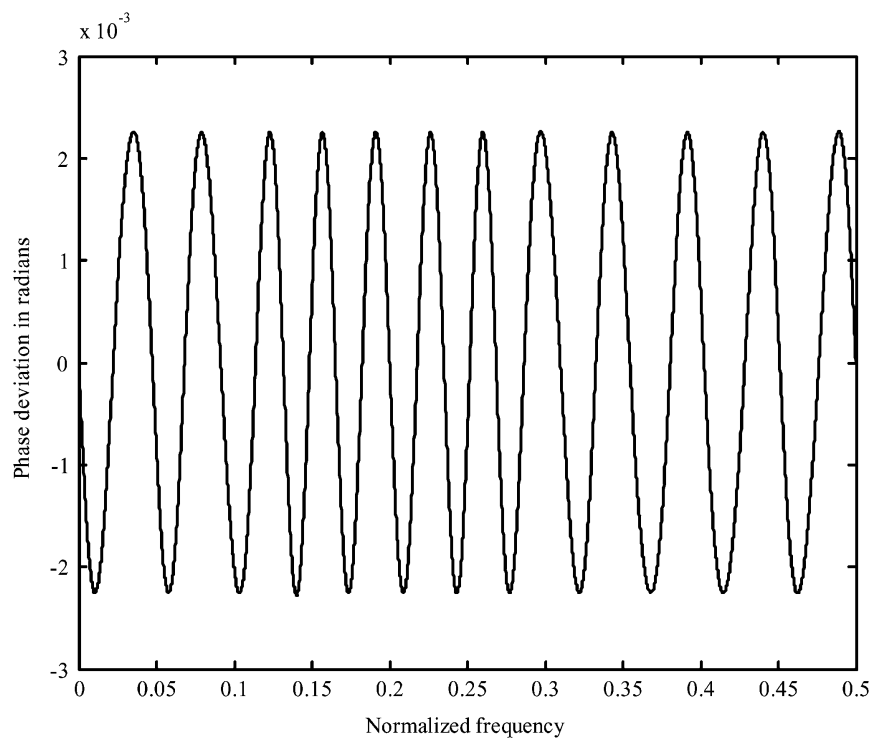

Fig. 7. Phase error response of the designed NDF bank for Example 1.

Next, we define the following two vectors:

$$
\mathbf{w}=\left(\mathbf{A D}_{\mathrm{x}}^{2} \mathbf{A}^{\mathrm{T}}\right)^{-1} \mathbf{A D}_{\mathrm{x}}^{2} \mathbf{c} \text { and } \mathbf{r}=\mathbf{c}-\mathbf{A}^{\mathrm{T}} \mathbf{w}
$$

where $\mathbf{w}$ represents the dual variable vector associated with the primal variable vector $\mathbf{x}$. Hence, the primal variable $\mathbf{x}^{k+1}$ at the $(k+1)$ th iteration is found by the following equation:

$$
\mathbf{x}^{k+1}=\mathbf{x}^{k}-\frac{\mu}{\eta} \mathbf{d}_{\mathrm{x}}
$$

where

$$
\text { and } \begin{aligned}
\mathbf{d}_{\mathrm{x}} & =\mathbf{D}_{\mathrm{x}}^{2} \mathbf{r} \\
\eta & =\max _{i}\left(\mathbf{e}_{\mathrm{i}}^{\mathrm{T}} \underline{\mathbf{c}}_{\mathrm{p}}\right)=\max _{i}\left(\mathbf{e}_{\mathrm{i}}^{\mathrm{T}} \mathbf{D}_{\mathrm{x}} \mathbf{r}\right) \\
& =\max _{i}\left(\mathbf{e}_{\mathrm{i}}^{\mathrm{T}} \mathbf{D}_{\mathrm{x}}^{2} \mathbf{r} / \mathbf{x}_{\mathrm{i}}\right)
\end{aligned}
$$

with $\mathrm{x}_{i}$ being the $i$ th entry of $\mathbf{x}$. 


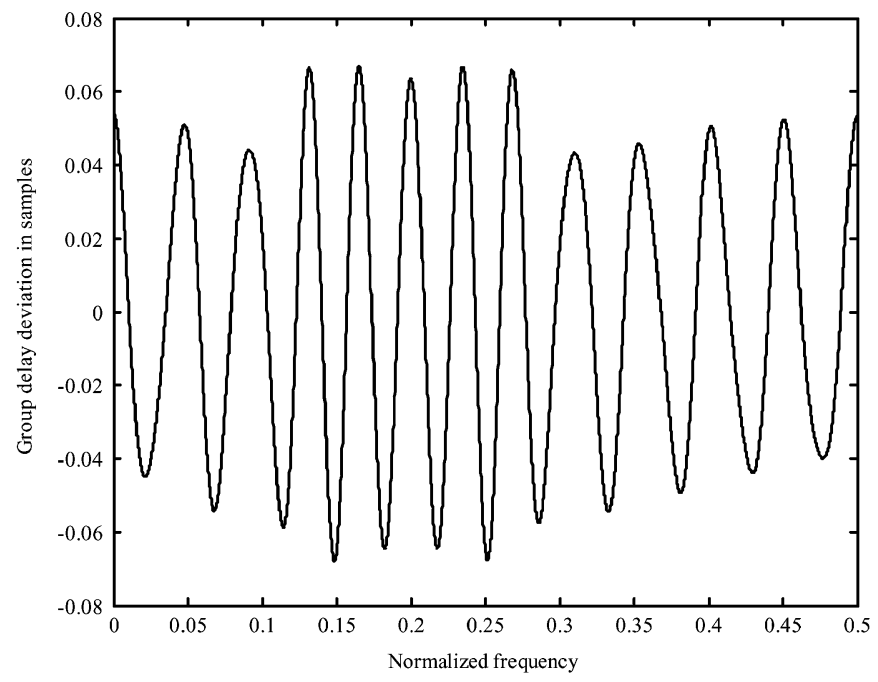

Fig. 8. Group delay deviation of the designed NDF bank for Example 1.

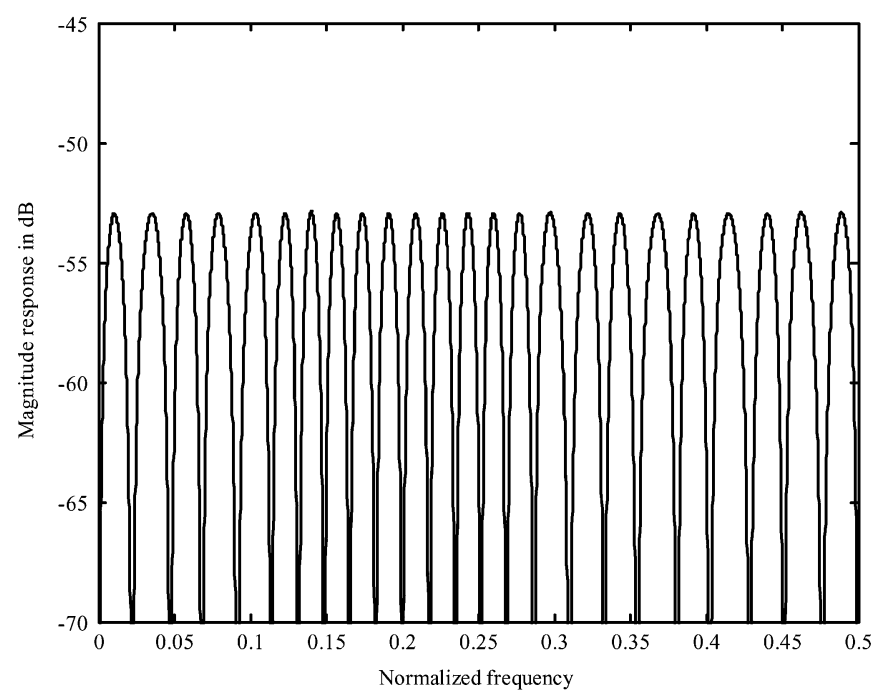

Fig. 9. Variation response of the design NDF bank response for Example 1.

\section{B. Determination of Initial Guess for the Filter Coefficient Vector}

To initiate the design process, we have to find an initial guess $\mathbf{a}_{i 0}$ for the filter coefficient vector $\mathbf{a}_{i}$ according to the nonlinear minimax algorithm of [20]. An appropriate manner is to minimize the squared value of the numerator of $\operatorname{Apx}_{i}\left(\mathbf{a}_{i}, \omega\right)$ given by (36), i.e., minimize

$$
\sum_{l=1}^{L}\left(\sum_{n=1}^{N_{i}} a_{i}(n)\left[\sin \left(n \omega_{l}\right)+P_{i}^{\prime}\left(\omega_{l}\right) \cos \left(n \omega_{l}\right)\right]+P_{i}^{\prime}\left(\omega_{l}\right)\right)^{2} .
$$

To solve this minimization problem, we use a simple approach as follows. Let $\mathbf{X}_{i}$ be a $S \times N_{i}$ matrix whose entries are given by

$$
\begin{aligned}
X_{i}(l, n)=\sin \left(n \omega_{l}\right) & +P_{i}^{\prime}\left(\omega_{l}\right) \cos \left(n \omega_{l}\right) \\
1 & \leq l \leq S, \quad 1 \leq n \leq N_{i}
\end{aligned}
$$

TABLE III

SIGNIFICANT DESIGN RESULTS FOR EXAMPLE 2

\begin{tabular}{c|c|c}
\hline & Proposed Technique & Technique of [11] \\
\hline Filter order & 31,32 & $13 / 14,17 / 17$ \\
\hline No. of coefficients & 63 & 63 \\
\hline PRE (dB) & $5.0145 \times 10^{-14}$ & 0.0176 \\
\hline MVGD (samples) & 0.0654 & 0.0703 \\
\hline NPSR $_{0}(\mathrm{~dB})$ & 34.10 & 40.88 \\
\hline $\mathrm{NPSR}_{1}(\mathrm{~dB})$ & 34.15 & 40.66 \\
\hline MVPGD $_{0}$ (samples) & 0.0277 & 0.0091 \\
\hline MVPGD $_{1}$ (samples) & 0.0327 & 0.0247 \\
\hline MVFBR $^{-3}$ & $1.58 \times 10^{-3}$ & $2.10 \times 10^{-3}$ \\
\hline No. of iterations & 5 & 41 \\
\hline
\end{tabular}

Then, it is easy to show that minimizing (59) yields the required initial guess $\mathbf{a}_{i 0}$ as follows:

$$
\mathbf{a}_{i 0}=\left(\mathbf{X}_{i}^{\mathrm{T}} \mathbf{X}_{i}\right)^{-1} \mathbf{X}_{i}^{\mathrm{T}} \mathbf{q}_{i}
$$

where the $S \times 1$ vector $\mathbf{q}_{i}$ has entries given by $q_{i}(l)=$ $-P_{i}^{\prime}\left(\omega_{l}\right), 1 \leq l \leq S$.

After finding the appropriate initial guess $\mathbf{a}_{i 0}$ and setting the initial filter coefficient increment to a zero vector, we present an iterative procedure based on the above presentation for computing the filter coefficient vector $\mathbf{a}_{i}$ during the design process.

\section{Iterative Procedure}

Step 1) Determine the design parameters: the orders $N_{1}$ and $N_{2}$, passband edge frequency $\omega_{p}$, and the stopband edge frequency $\omega_{s}$. Find an initial guess $\mathbf{a}_{i 0}$ for the filter coefficient vector $\mathbf{a}_{i}=\left[a_{i}(1), a_{i}(2), \ldots, a_{i}\left(N_{i}\right)\right]^{\mathrm{T}}, i=1,2$ from (61) and set the iteration number $k=0$.

Step 2) Perform a test for stopping the iteration process. We set a stopping criterion as follows: If $\left|V_{k-1}-V_{k}\right| /\left|V_{k-1}\right| \leqq$ $\kappa_{1}$, then the design process is terminated and the obtained filter coefficient vector $\mathbf{a}_{i k}$ contains the designed filter coefficients, where $V_{k}=\gamma_{1}\left\|\operatorname{Apx}_{1}\left(\mathbf{a}_{1 k}, \omega_{l}\right)\right\|+$ $\gamma_{2}\left\|\operatorname{Apx}_{2}\left(\mathbf{a}_{2 k}, \omega_{l}\right)\right\|+\gamma\left\|\operatorname{Apx}_{\mathrm{T}}\left(\mathbf{a}_{1 k}, \mathbf{a}_{2 k}, \omega_{l}\right)\right\|$, and $\kappa_{1}$ is a preset small positive number. Otherwise, go to Step 3.

Step 3) Calculate the increment $\delta \mathbf{a}_{i}$ of the filter coefficient vector $\mathbf{a}_{i k}=\left[a_{i k}(1), a_{i k}(2), \ldots, a_{i k}\left(N_{i}\right)\right]^{\mathrm{T}}$ at the $k$ th iteration to minimize (43). Utilizing the PAS algorithm presented above to solve the standard LP problem of (48), we perform the following iterative procedure.

3.1) Choose an initial guess $\mathrm{x}^{0}$ that satisfies the equality constraints $\mathbf{A}^{\mathrm{T}} \mathbf{x}^{0}=\mathbf{b}$ and $\mathbf{x}^{0}>0$ of (48). We can simply choose $x^{0}=$ $\left[\begin{array}{llllll}\mathbf{x}_{1}^{\mathrm{T}} & \mathbf{x}_{2}^{\mathrm{T}} & \mathbf{x}_{3}^{\mathrm{T}} & \mathbf{x}_{4}^{\mathrm{T}} & \mathbf{x}_{5}^{\mathrm{T}} & \mathbf{x}_{6}^{\mathrm{T}}\end{array}\right]^{\mathrm{T}}$, where $\mathbf{x}_{1}=\mathbf{x}_{2}=$ $\gamma_{1} \mathbf{1} /(2 S), \mathbf{x}_{3}=\mathbf{x}_{4}=\gamma_{2} \mathbf{1} /(2 S), \mathbf{x}_{5}=\mathbf{x}_{6}=$ $\gamma \mathbf{1} /(2 S)$, and each of $\mathbf{x}_{j}$ has entries given by $x_{j}(l), j=1,2, \ldots, 6, l=1,2, \ldots, S$. Set the iteration number $m=0$.

3.2) At the $m$ th iteration, compute $\mathbf{w}=$ $\left[\begin{array}{lllll}\delta \mathbf{a}_{1}^{\mathrm{T}} & \delta \mathbf{a}_{2}^{\mathrm{T}} & \xi_{1} & \xi_{2} & \xi_{T}\end{array}\right]^{\mathrm{T}}=\left(\mathbf{A D}_{\mathbf{x}}^{2} \mathbf{A}^{\mathrm{T}}\right)^{-1} \mathbf{A D}_{\mathbf{x}}^{2} \mathbf{c}$ with $\mathbf{D}_{\mathbf{x}}=\operatorname{diag}\left(\mathbf{x}^{m}\right)$ according to the following process. 
3.2.1) Construct six vectors $\mathbf{p}_{1}, \mathbf{p}_{2}, \mathbf{p}_{3}, \mathbf{m}_{1}, \mathbf{m}_{2}, \mathbf{m}_{3}$. The $l$ th entry for each of them is, respectively, given by

$$
\begin{aligned}
p_{1}(l)=x_{1}^{2}(l)+x_{2}^{2}(l), & m_{1}(l)=x_{1}^{2}(l)-x_{2}^{2}(l), \\
p_{2}(l)=x_{3}^{2}(l)+x_{4}^{2}(l), & m_{2}(l)=x_{3}^{2}(l)-x_{4}^{2}(l) \\
p_{3}(l)=x_{5}^{2}(l)+x_{6}^{2}(l), & m_{3}(l)=x_{5}^{2}(l)-x_{6}^{2}(l) \\
& l=1,2, \ldots, S .
\end{aligned}
$$

3.2.2) Compute $\mathbf{Q}_{i}=\operatorname{diag}\left(\mathbf{p}_{i}^{m}\right)-\mathbf{m}_{i} \mathbf{m}_{i}^{\mathrm{T}} / g_{i}$, where $g_{i}=\sum_{l=1}^{S} p_{i}(l)$, for $i=1,2,3$.

3.2.3)

$$
\text { Compute }
$$

$$
\begin{aligned}
{\left[\delta \mathbf{a}_{1 m}^{\mathrm{T}} \delta \mathbf{a}_{2 m}^{\mathrm{T}}\right]^{\mathrm{T}} } & \\
= & {\left[\begin{array}{cc}
\mathbf{E}_{1}^{\mathrm{T}} \mathbf{Q}_{1} \mathbf{E}_{1}+\mathbf{E}_{\mathrm{T} 1}^{\mathrm{T}} \mathbf{Q}_{3} \mathbf{E}_{\mathrm{T} 1} & \mathbf{E}_{\mathrm{T} 1}^{\mathrm{T}} \mathbf{Q}_{3} \mathbf{E}_{\mathrm{T} 2} \\
\mathbf{E}_{\mathrm{T} 2}^{\mathrm{T}} \mathbf{Q}_{3} \mathbf{E}_{\mathrm{T} 1} & \mathbf{E}_{2}^{\mathrm{T}} \mathbf{Q}_{2} \mathbf{E}_{2}+\mathbf{E}_{\mathrm{T} 2}^{\mathrm{T}} \mathbf{Q}_{3} \mathbf{E}_{\mathrm{T} 2}
\end{array}\right]^{-1} } \\
& \times\left[\begin{array}{l}
\mathbf{E}_{1}^{\mathrm{T}} \mathbf{Q}_{1} \mathbf{s}_{1}+\mathbf{E}_{\mathrm{T} 1}^{\mathrm{T}} \mathbf{Q}_{3} \mathbf{S}_{\mathrm{T}} \\
\mathbf{E}_{2}^{\mathrm{T}} \mathbf{Q}_{2} \mathbf{s}_{2}+\mathbf{E}_{\mathrm{T} 2}^{\mathrm{T}} \mathbf{Q}_{3} \mathbf{s}_{\mathrm{T}}
\end{array}\right] \\
\xi_{1}= & \left(\mathbf{m}_{1}^{\mathrm{T}}\left(\mathbf{E}_{1} \delta \mathbf{a}_{1 m}-\mathbf{s}_{1}\right)\right) / g_{1} \\
\xi_{2}= & \left(\mathbf{m}_{2}^{\mathrm{T}}\left(\mathbf{E}_{2} \delta \mathbf{a}_{2 m}-\mathbf{s}_{2}\right)\right) / g_{2} \\
\xi_{\mathrm{T}}= & \left(\mathbf{m}_{3}^{\mathrm{T}}\left(\mathbf{E}_{\mathrm{T} 1} \delta \mathbf{a}_{1 m}+\mathbf{E}_{\mathrm{T} 2} \delta \mathbf{a}_{2 m}-\mathbf{s}_{T}\right)\right) / g_{3} .
\end{aligned}
$$

$$
\begin{aligned}
& \text { Compute the vector } \mathbf{r}=\mathbf{c}-\mathbf{A}^{\mathrm{T}} \mathbf{w}= \\
& {\left[\mathbf{r}_{1}^{\mathrm{T}} \mathbf{r}_{2}^{\mathrm{T}} \mathbf{r}_{3}^{\mathrm{T}} \mathbf{r}_{4}^{\mathrm{T}} \mathbf{r}_{5}^{\mathrm{T}} \mathbf{r}_{6}^{\mathrm{T}}\right]^{\mathrm{T}} \text { as follows: Con- }} \\
& \text { struct } \mathbf{f}_{1}=\mathbf{E}_{1} \delta \mathbf{a}_{1 m} ; \mathbf{f}_{2}=\mathbf{E}_{2} \delta \mathbf{a}_{2 m} ; \mathbf{f}_{\mathrm{T}}= \\
& \mathbf{E}_{\mathrm{T} 1} \delta \mathbf{a}_{1 m}+\mathbf{E}_{\mathrm{T} 1} \delta \mathbf{a}_{2 m} . \text { Then, set } \mathbf{r}_{1}= \\
& \mathbf{s}_{1}+\xi_{1} \mathbf{1}-\mathbf{f}_{1} ; \mathbf{r}_{2}=-\mathbf{s}_{1}+\xi_{1} \mathbf{1}+\mathbf{f}_{1} ; \\
& \mathbf{r}_{3}=\mathbf{s}_{2}+\xi_{2} \mathbf{1}-\mathbf{f}_{2} ; \mathbf{r}_{4}=-\mathbf{s}_{2}+\xi_{2} \mathbf{1}+\mathbf{f}_{2} ; \\
& \mathbf{r}_{5}=\mathbf{s}_{\mathrm{T}}+\xi_{\mathrm{T}} \mathbf{1}-\mathbf{f}_{\mathrm{T}} ; \mathbf{r}_{6}=-\mathbf{s}_{\mathrm{T}}+\xi_{\mathrm{T}} \mathbf{1}-\mathbf{f}_{\mathrm{T}} .
\end{aligned}
$$

Step 4) Compute $\xi_{1} / \max \left\{\left|\mathbf{E}_{1} \delta \mathbf{a}_{1 m}-\mathbf{s}_{1}\right|\right\}, \xi_{2} /$ $\max \left\{\left|\mathbf{E}_{2} \delta \mathbf{a}_{2 m}-\mathbf{s}_{2}\right|\right\}, \quad$ and $\xi_{\mathrm{T}} / \max \left\{\mid \mathbf{E}_{\mathrm{T} 1} \delta \mathbf{a}_{1 m}+\right.$ $\left.\mathbf{E}_{\mathrm{T} 2} \delta \mathbf{a}_{2 m}-\mathbf{s}_{\mathrm{T}} \mid\right\}$. Set the obtained $\delta \mathbf{a}_{\text {im }}$ equal to $\delta \mathbf{a}_{i k}, i=1,2$, and go to Step 7 if $\xi_{1} / \max \left\{\left|\mathbf{E}_{1} \delta \mathbf{a}_{1 m}-\mathbf{s}_{1}\right|\right\} \geqq$ $\kappa_{2}, \xi_{2} / \max \left\{\left|\mathbf{E}_{2} \delta \mathbf{a}_{2 m} \quad-\mathbf{s}_{2}\right|\right\} \quad \geqq \quad \kappa_{3}, \quad$ and $\xi_{\mathrm{T}} / \max \left\{\left|\mathbf{E}_{\mathrm{T} 1} \delta \mathbf{a}_{1 m}+\mathbf{E}_{\mathrm{T} 2} \delta \mathbf{a}_{2 m}-\mathbf{s}_{\mathrm{T}}\right|\right\} \geqq \kappa_{4}$, where $\kappa_{2}, \kappa_{3}$, and $\kappa_{4}$ are preset positive numbers less than one. Otherwise, go to Step 5.

Step 5) Compute $\mathbf{d}_{\mathrm{x}}=\mathbf{D}_{\mathrm{x}}^{2} \mathbf{r}=\mathbf{D}_{\mathrm{x}}^{2}\left(\mathbf{c}-\mathbf{A}^{\mathrm{T}} \mathbf{w}\right)$ according to the following process.

5.1) Compute $\mathbf{d}_{1}=\mathbf{D}_{1}^{2} \mathbf{r}_{1}, \mathbf{d}_{2}=\mathbf{D}_{2}^{2} \mathbf{r}_{2}, \mathbf{d}_{3}=$ $\mathbf{D}_{3}^{2} \mathbf{r}_{3}, \mathbf{d}_{4}=\mathbf{D}_{4}^{2} \mathbf{r}_{4}, \mathbf{d}_{5}=\mathbf{D}_{5}^{2} \mathbf{r}_{5}, \mathbf{d}_{6}=\mathbf{D}_{6}^{2} \mathbf{r}_{6}$, where $\mathbf{D}_{i}=\operatorname{diag}\left(\mathbf{x}_{i}^{m}\right)$, for $i=1,2, \ldots, 6$.

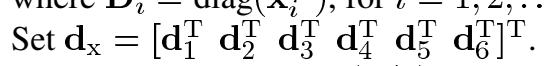

Step 6) Compute the step size $(\mu / \eta)$ from (58) with $\mu=0.97$, and update the primal variable vector according to $\mathbf{x}^{m+1}=\mathbf{x}^{m}-(\mu / \eta) \mathbf{d}_{x}$. Then, set $m=m+1$, and go to Step 3.2.

Step 7) Use the obtained optimal solution $\delta \mathbf{a}_{i k}$ to find the best increment such that

$$
\begin{array}{r}
\gamma_{1}\left\|\operatorname{Apx}_{1}\left(\mathbf{a}_{1 k}+\beta \delta \mathbf{a}_{1 k}, \omega_{l}\right)\right\|+\gamma_{2}\left\|\operatorname{Apx}_{2}\left(\mathbf{a}_{2 k}+\beta \delta \mathbf{a}_{2 k}, \omega_{l}\right)\right\| \\
+\gamma\left\|\operatorname{Apx}_{\mathrm{T}}\left(\mathbf{a}_{1 k}+\beta \delta \mathbf{a}_{1 k}, \mathbf{a}_{2 k}+\beta \delta \mathbf{a}_{2 k}, \omega_{l}\right)\right\| \\
\forall \beta \geqq 0
\end{array}
$$

is minimized. We adopt the Nelder and Mead simplex algorithm [25] to perform the line search for finding the best value of $\beta$. Let the best value of $\beta$ be $\beta_{k}$. We update the filter co- efficient vector according to $\mathbf{a}_{i(k+1)}=\mathbf{a}_{i k}+\beta_{k} \delta \mathbf{a}_{i k}$. Then, set $k=k+1$, and go to Step 2 .

\section{Simulation Results}

In this section, we present simulation results of designing two-channel linear-phase NDF banks with the proposed real IIR DAFs structure for illustration and comparison. These designs were performed on a personal computer with Pentium-IV CPU using MATLAB programming language. For comparison, the design results of using the proposed technique and the techniques presented by [11] are also presented. The performance for each of the designed IIR NDF banks is evaluated in terms of the perfect reconstruction error (PRE), the normalized peak stopband ripple of $H_{i}(z)\left(\mathrm{NPSR}_{i}\right)$, the maximal variation of passband group delay of $H_{i}(z)\left(\mathrm{MVPGD}_{i}\right)$, the maximal variation of the group delay (MVGD) in $\hat{T}\left(e^{j \omega}\right)$, and the maximal variation of the filter-bank response (MVFBR). They are defined as follows:

$$
\begin{aligned}
\text { PRE }= & \max \left\{\left|20 \log _{10}\right| \hat{T}\left(\omega_{l}\right)||\right\}, \quad \text { for } \omega_{l} \in[0, \pi] \\
\operatorname{NPSR}_{0}= & -20 \log _{10}\left(\max _{\omega_{l} \in\left[\omega_{s}, \pi\right]} \frac{\left|H_{0}\left(e^{j \omega_{l}}\right)\right|}{\sqrt{L L_{0}}}\right)(\mathrm{dB}) \\
\mathrm{NPSR}_{1}= & -20 \log _{10}\left(\max _{\omega_{l} \in\left[0, \omega_{p}\right]} \frac{\left|H_{1}\left(e^{j \omega_{l}}\right)\right|}{\sqrt{L L_{1}}}\right)(\mathrm{dB}) \\
\operatorname{MVPGD}_{0}= & \max _{\omega_{l} \in\left[0, \omega_{p}\right]} \mid \operatorname{GD}\left\{H_{0}\left(e^{j \omega_{l}}\right)\right\} \\
& -\frac{1}{2}\left(N_{1}+N_{2}\right) \mid(\text { samples }) \\
\operatorname{MVPGD}_{1}= & \max _{\omega_{l} \in\left[\omega_{s}, \pi\right]} \mid \operatorname{GD}\left\{H_{1}\left(e^{j \omega_{l}}\right)\right\} \\
& -\frac{1}{2}\left(N_{1}+N_{2}\right) \mid(\text { samples }) \\
\operatorname{MVGD}= & \max _{\omega_{l} \in[0, \pi]} \mid \operatorname{GD}\left\{\hat{T}\left(e^{j \omega_{l}}\right)\right\} \\
& -\left(N_{1}+N_{2}\right) \mid(\text { samples }) \\
\operatorname{MVFBR}= & \max _{\omega_{l} \in[0, \pi]}\left|\hat{T}\left(e^{j \omega_{l}}\right)-e^{-j\left(N_{1}+N_{2}\right) \omega_{l}}\right|
\end{aligned}
$$

where $\mathrm{GD}\{x\}$ denotes the group delay of $x$. The performance for each of the NDF banks designed by using the technique of [11] is evaluated based on the filter coefficients presented in [11].

Example 1: This example is the similar to that given by [11, Ex. 1]. We use the same specifications for this design: the real IIR DAFs $A_{1}(z)$ and $A_{2}(z)$ with orders $N_{1}$ and $N_{2}$ equal to 21 and 22, respectively, the lowpass analysis filter $H_{0}(\mathrm{z})$ with a passband edge frequency $\omega_{p}=0.3 \pi$ and a stopband edge frequency $\omega_{s}=0.5 \pi$. The parameters used for this design are listed as follows: $L_{0}=2, L_{1}=3 ; \kappa_{1}=10^{-11}, \kappa_{2}=$ $\kappa_{3}=\kappa_{4}=1-10^{-5} ; \gamma_{1}=\gamma_{2}=40, \gamma=100$; and $S_{1}=$ $100, S_{2}=72, S_{3}=130$. The significant design results, namely, PRE, NPSR, MVPR, MVGD, and MVFBR designed by using the technique of [11] and the proposed technique are shown in Table I for comparison. Table II lists the filter coefficients obtained after six iterations for $A_{1}(z)$ and $A_{2}(z)$ by using the proposed technique. The corresponding magnitude responses of the 
TABLE IV

DESIGNED FILTER COEFFICIENTS FOR EXAMPLE 2

\begin{tabular}{|c|c|c|}
\hline$n$ & $a_{1}(n)$ & $a_{2}(n)$ \\
\hline 0 & 1.00000000000000 & 1.00000000000000 \\
\hline 1 & 0.40028247713820 & -0.40032121293172 \\
\hline 2 & 0.15423987572849 & 0.00605895028584 \\
\hline 3 & -0.00646042103950 & 0.06580391863711 \\
\hline 4 & -0.09418993240059 & 0.06432964720258 \\
\hline 5 & -0.11533127485475 & 0.04177298107076 \\
\hline 6 & -0.08887227036703 & 0.01708155101606 \\
\hline 7 & -0.04146343802359 & -0.00005238263035 \\
\hline 8 & 0.00191771750421 & -0.00665690808421 \\
\hline 9 & 0.02645787367772 & -0.00545184397568 \\
\hline 10 & 0.03012127974346 & -0.00146651159786 \\
\hline 11 & 0.02037294949627 & 0.00112280900881 \\
\hline 12 & 0.00784430196404 & 0.00062018877514 \\
\hline 13 & 0.00030682841581 & -0.00206629119141 \\
\hline 14 & -0.00029953152382 & -0.00467137309649 \\
\hline 15 & 0.00295909196495 & -0.00526050549725 \\
\hline 16 & 0.00539022155031 & -0.00344223682094 \\
\hline 17 & 0.00395880605195 & -0.00022597252693 \\
\hline 18 & -0.00111865416043 & 0.00272734372372 \\
\hline 19 & -0.00709464946488 & 0.00417799277480 \\
\hline 20 & -0.01072386476082 & 0.00384159836781 \\
\hline 21 & -0.01021937042140 & 0.00231976640520 \\
\hline 22 & -0.00620016782550 & 0.00072596537172 \\
\hline 23 & -0.00098938454323 & -0.00013377710659 \\
\hline 24 & 0.00272048111328 & 0.00007516788480 \\
\hline 25 & 0.00343330572491 & 0.00095138844690 \\
\hline 26 & 0.00153902803881 & 0.00171661043598 \\
\hline 27 & -0.00099285234042 & 0.00156180072171 \\
\hline 28 & -0.00163034617467 & -0.00003922543466 \\
\hline 29 & 0.00102345265330 & -0.00264243948991 \\
\hline 30 & 0.00501588286110 & -0.00382066697890 \\
\hline 31 & -0.00309714050884 & 0.00774535238295 \\
\hline 32 & & -0.00384153443848 \\
\hline
\end{tabular}

designed $H_{i}\left(e^{j \omega}\right)$ are shown in Fig. 6. The resulting phase error and group delay deviation of the designed NDF bank are depicted in Figs. 7 and 8, respectively. Fig. 9 plots the variation of the designed filterbank response. As to the stability of the designed IIR DAFs $A_{1}(z)$ and $A_{2}(z)$, we find that the values of $\theta_{i}(\pi)-\theta_{i}(0)$ are about $-21 \pi$ and $-22 \pi$ for $i=1$ and 2 , respectively. This indicates that both the designed IIR DAFs $A_{1}(z)$ and
$A_{2}(z)$ are stable according to the stability constraints shown in Section II-B. From the simulation results, we note that the proposed technique provides very satisfactory design results as compared with the results of [11].

Example 2: This example is the similar to that given by [11, Ex. 2]. We use the same specifications for this design: the real IIR DAFs $A_{1}(z)$ and $A_{2}(z)$ with orders $N_{1}$ and $N_{2}$ equal to 


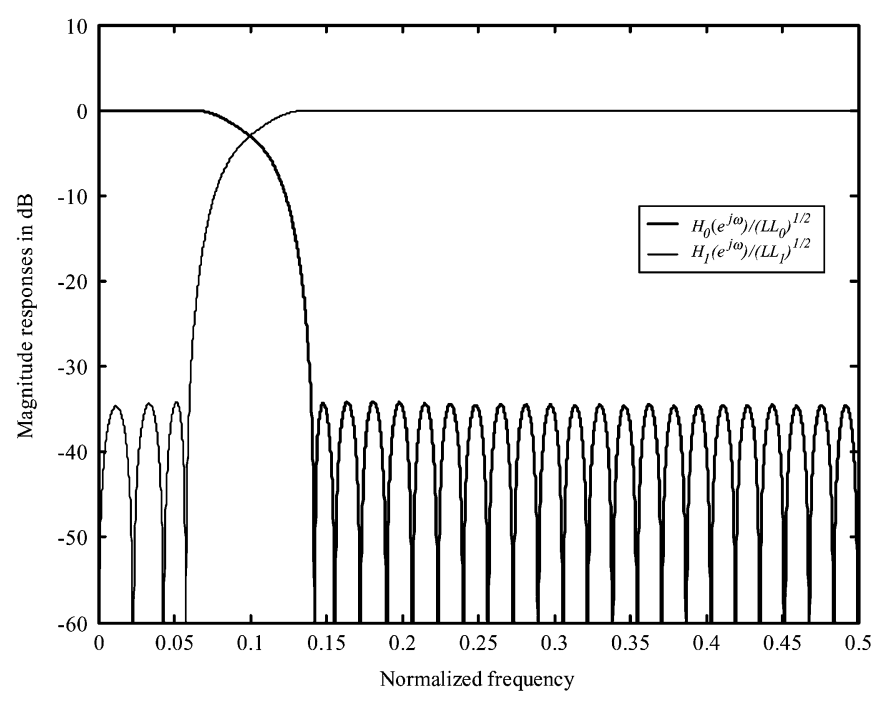

Fig. 10. Magnitude responses of the designed analysis filters for Example 2.

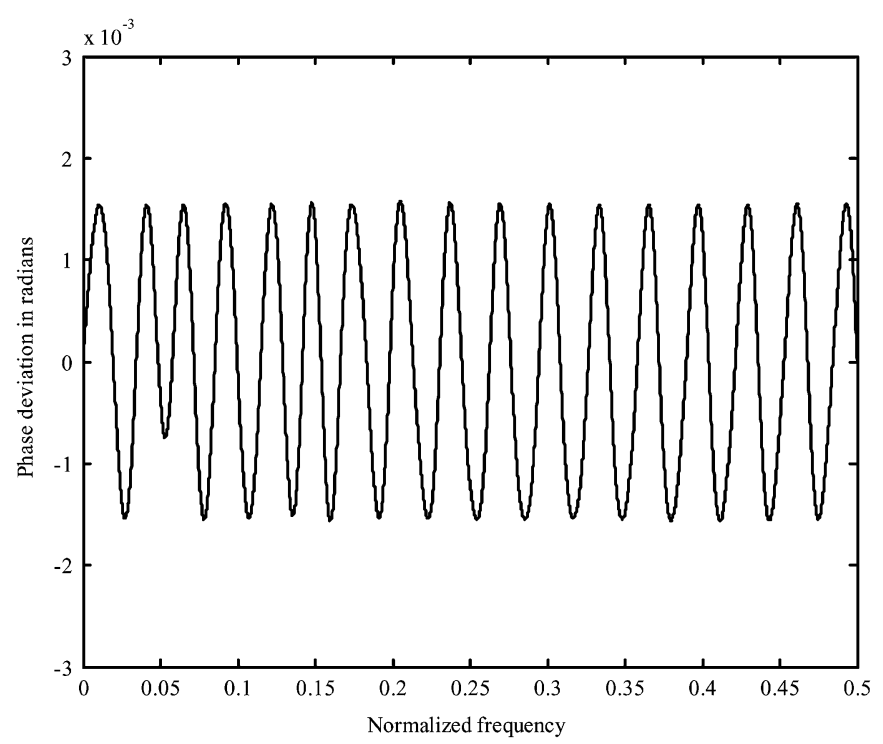

Fig. 11. Phase error response of the designed NDF bank for Example 2.

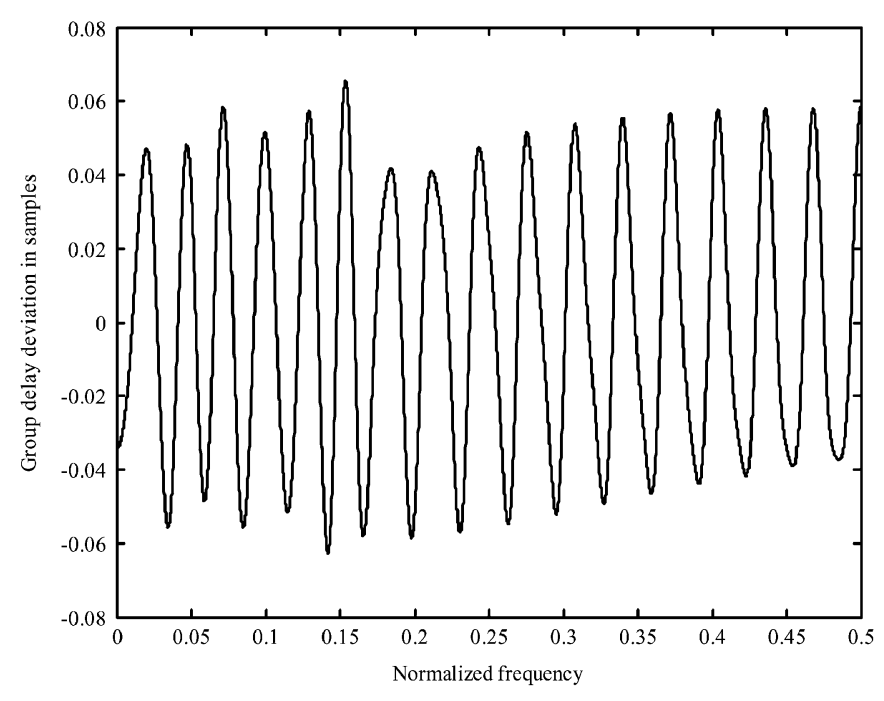

Fig. 12. Group delay deviation of the designed NDF bank for Example 2.

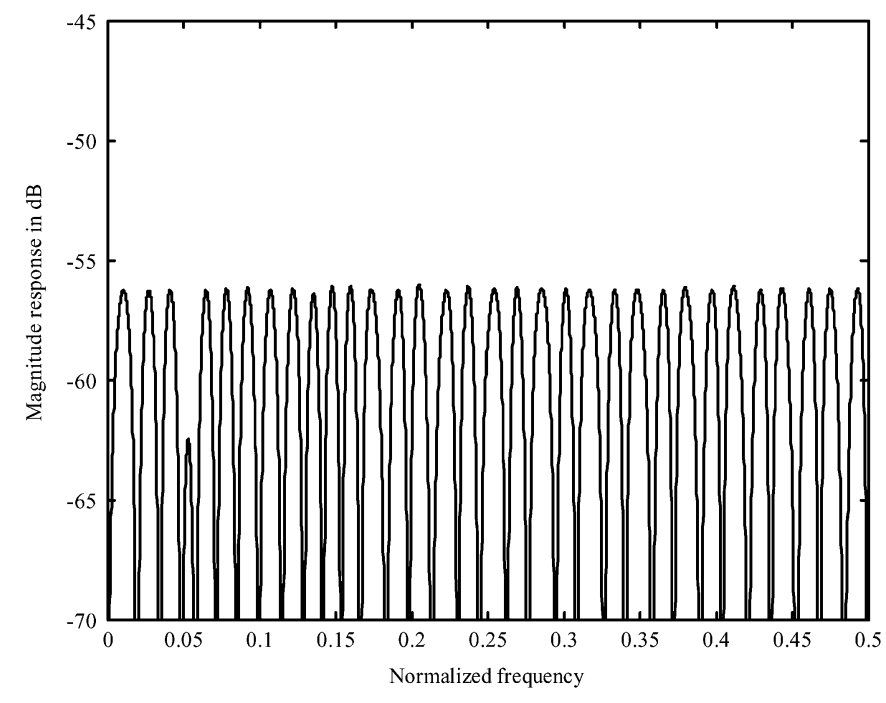

Fig. 13. Variation response of the design NDF bank response for Example 2.

31 and 32, respectively, the lowpass analysis filter $H_{0}(\mathrm{z})$ with a passband edge frequency $\omega_{p}=0.12 \pi$ and a stopband edge frequency $\omega_{s}=0.28 \pi$. The parameters used for this design are listed as follows: $L_{0}=1, L_{1}=4 ; \kappa_{1}=10^{-12}, \kappa_{2}=$ $\kappa_{3}=\kappa_{4}=1-10^{-5} ; \gamma_{1}=\gamma_{2}=61, \gamma=100$; and $S_{1}=$ $80, S_{2}=52, S_{3}=170$. The significant design results of using the technique of [11] and the proposed technique are shown in Table III for comparison. Table IV lists the filter coefficients obtained after five iterations for $A_{1}(z)$ and $A_{2}(z)$, respectively, by using the proposed technique. The corresponding magnitude responses of the designed $H_{i}\left(e^{j \omega}\right)$ are shown in Fig. 10. The resulting phase error and group delay deviation of the designed NDF bank are depicted in Figs. 11 and 12, respectively. Fig. 13 plots the variation of the designed filterbank response. As to the stability of the designed IIR DAFs $A_{1}(z)$ and $A_{2}(z)$, we find that the values of $\theta_{i}(\pi)-\theta_{i}(0)$ are about $-31 \pi$ and $-32 \pi$ for $i=1$ and 2 , respectively. This indicates that both the designed IIR DAFs $A_{1}(z)$ and $A_{2}(z)$ are stable according to the stability constraints shown in Section II-B. Again, we note from the simulation results that the proposed technique provides very satisfactory performance.

\section{CONCLUSION}

This paper has presented a technique for the design of twochannel linear-phase nonuniform-division filter (NDF) banks based on real infinite impulse response (IIR) digital allpass filters (DAFs). First, the theory of two-channel NDF bank structures using two IIR DAFs $A_{1}(z)$ and $A_{2}(z)$ was developed. The resulting two-channel NDF bank structure possesses perfect magnitude response, i.e., there is no magnitude distortion, and its phase is equal to the sum of the phases of $A_{1}\left(e^{j \omega}\right)$ and $A_{2}\left(e^{j \omega}\right)$. The design problem was then formulated as a nonlinear optimization problem of an appropriate objective function for the phase response using the minimax $\left(L_{\infty}\right)$ error criteria. A nonlinear minimax algorithm is used to generate a sequence of linear Chebyshev approximation problems. Solving each of the linear Chebyshev approximations provides the required increment for updating the filter coefficients during each iteration. 
This leads to that the linear Chebyshev approximation of a desired response from a linear subspace related to the objective function can be easily solved by using a variant of Karmarkar's algorithm. Computer simulations have been provided for confirmation and comparison.

\section{REFERENCES}

[1] R. E. Crochiere, "Digital signal processor: Sub-band coding," Bell Syst. Tech. J., vol. 60, pp. 1633-1653, 1981.

[2] M. G. Bellanger and J. L. Daguet, "TDM-FDM transmultiplexer: Digital polyphase and FFT," IEEE Trans. Commun., vol. COM-22, pp. 1199-1204, 1974.

[3] P. Vary and U. Heute, "A short-time spectrum analyzer with polyphase network and DFT," Signal Process., vol. 2, pp. 55-65, 1980.

[4] J. W. Woods and S. D. O'Neil, "Subband coding of images," IEEE Trans. Acoust., Speech, Signal Processing, vol. 34, pp. 1278-1288, Oct. 1986.

[5] J. Kovacevic, "Filter banks and wavelets: Extensions and applications," Ph.D. Dissertation, Columbia Univ., New York, Oct. 1991.

[6] K. Nayebi, T. P. Barnwell, and M. J. T. Smith, "Nonuniform filter banks: A reconstruction and design theory," IEEE Trans. Signal Processing, vol. 41, pp. 1114-1127, Mar. 1993.

[7] S. Wada, "Design of nonuniform division multirate FIR filter banks," IEEE Trans. Circuits Syst. II, vol. 42, pp. 115-121, Feb. 1995.

[8] J.-H. Lee and S.-C. Huang, "Design of two-channel nonuniform-division maximally decimated filter banks using $L_{1}$ criteria," Proc. Inst. Elect. Eng., Vision, Image, Signal Process., vol. 143, pp. 79-83, 1996.

[9] J.-H. Lee and W.-H. Chung, "Design of two-channel low-delay IIR nonuniform-division filter banks using $L_{1}$ error criteria," Proc. Inst. Elect. Eng., Vision, Image, Signal Process., to be published.

[10] J.-H. Lee and D.-C. Tang, "Optimal design of two-channel nonuniformdivision FIR filter banks with $-1,0$, and +1 coefficients," IEEE Trans. Signal Processing, vol. 47, pp. 422-432, Feb. 1999.

[11] J.-H. Lee and I.-C. Niu, "Design of two-channel IIR nonuniform-division filter banks with arbitrary group delay," Proc. Inst. Elect. Eng., Vision, Image, Signal Process., vol. 147, pp. 534-542, Dec. 2000.

[12] J.-H. Lee and D.-C. Tang, "Minimax design of two-channel nonuniform-division FIR filter banks," in Proc. Inst. Elect. Eng., Vision, Image, Signal Process., vol. 145, 2, Apr. 1998, pp. 88-96.

[13] - "Minimax design of two-channel nonuniform-division FIR filter banks with $-1,0$, and +1 coefficients," IEEE Trans. Circuits Syst. I, vol. 46, pp. 1184-1190, Oct. 1999.

[14] F. Argenti, V. Cappellini, A. Sciorpes, and A. N. Venetsanopoulos, "Design of IIR linear-phase QMF banks based on complex allpass sections," IEEE Trans. Signal Processing, vol. 44, pp. 1262-1267, May 1996.

[15] C.-K. Lu, M. Anderson, and S. Summerfield, "Design of approximately linear-phase allpass based QMF banks," in Proc. Int. Symp. Digital Signal Process., London, U.K., 1996, pp. 56-61.

[16] S. Summerfield and C.-K. Lu, "Design and VLSI implementation of multirate filter banks based on approximately linear phase allpass sections," in Proc. Int. Symp. Circuits Syst., Monterey, CA, 1998, pp. 413-416.

[17] S. S. Lawson and A. Klouche-Djedid, "Technique for design of two-channel approximately linear phase QMF bank and its application to image compression," Proc. Inst. Elect. Eng., Vision, Image, Signal Process., vol. 148, pp. 85-92, 2001.
[18] S. S. Lawson, "Design of IIR-based wavelet filter banks and their application to image coding," in Proc. IEE Seminar Time-Scale Time-Freq. Anal. Applicat., 2000, pp. 7/1-7/6.

[19] P. P. Vaidyanathan, P. A. Regalia, and S. K. Mitra, "Design of doublycomplementary IIR digital filters using a single complex allpass filter, with multirate applications," IEEE Trans. Circuits Syst., vol. CAS-34, pp. 378-388, Apr. 1987.

[20] M. R. Osborne and G. A. Watson, "An algorithm for minimax approximation in the nonlinear case," Comput. J., vol. 12, pp. 63-68, 1969.

[21] I. Adler, N. Karmarkar, M. G. C. Resende, and G. Veiga, "An implementation of Karmarkar's algorithm for linear programming," Math. Programming, vol. 44, pp. 297-335, 1989.

[22] M. Ikehara, M. Funaishi, and H. Kuroda, "Design of complex all-pass networks using Remez algorithm," IEEE Trans. Circuits Syst. II, vol. 39, pp. 549-556, Aug. 1992.

[23] P. P. Vaidyanathan, Multirate Systems and Filter Banks. Englewood Cliffs, NJ: Prentice-Hall, 1992.

[24] C. H. Papadimitriou and K. Steiglitz, Combinatorial Optimization: Algorithms and Complexity. Englewood Cliffs, NJ: Prentice-Hall, 1982.

[25] J. A. Nelder and R. Meade, "A simplex method for function minimization," Comput. J., vol. 7, pp. 308-313, 1965.

[26] E. W. Cheney, Introduction to Approximation Theory. New York: McGraw-Hill, 1966.

Ju-Hong Lee was born in I-Lan, Taiwan, R.O.C., on December 7, 1952. He received the B.S. degree from the National Cheng-Kung University, Tainan, Taiwan, in 1975, the M.S. degree from the National Taiwan University (NTU), Taipei, Taiwan, in 1977, and the Ph.D. degree from Rensselaer Polytechnic Institute (RPI), Troy, NY, in 1984, all in electrical engineering.

From September 1980 to July 1984, he was a Research Assistant and was involved in research on multidimensional recursive digital filtering with the Department of Electrical, Computer, and Systems Engineering at RPI. From August 1984 to July 1986, he was a Visiting Associate Professor and later in August 1986 became an Associate Professor with the Department of Electrical Engineering, NTU, where he has been a Professor since August 1989. He was appointed Visiting Professor with the Department of Computer Science and Electrical Engineering, University of Maryland, Baltimore, during a sabbatical leave in 1996. His current research interests include multidimensional digital signal processing, image processing, detection, and estimation theory, analysis and processing of joint vibration signals for the diagnosis of cartilage pathology, and adaptive signal processing and its applications in communications.

Dr. Lee received Outstanding Research Awards from the National Science Council (NSC) of Taiwan in 1988, 1989, and 1991 to 1994 and Distinguished Research Awards from the NSC from 1998 to 2003.

Yuan-Hau Yang was born in Yun-Lin, Taiwan, R.O.C., on March 5, 1977. He received the B.S. degree in electrical engineering from the National Chiao-Tung University, Hsin-Chu, Taiwan, in 2000 and the M.S. degree in electrical engineering from the National Taiwan University, Taipei, Taiwan, in 2003.

His current research interests include digital signal processing and its applications. 